\title{
A DUALITY THEORY FOR NON-CONVEX PROBLEMS IN THE CALCULUS OF VARIATIONS
}

\author{
GUY BOUCHITTÉ, ILARIA FRAGALÀ
}

\begin{abstract}
We present a new duality theory for non-convex variational problems, under possibly mixed Dirichlet and Neumann boundary conditions. The dual problem reads nicely as a linear programming problem, and our main result states that there is no duality gap. Further, we provide necessary and sufficient optimality conditions, and we show that our duality principle can be reformulated as a min-max result which is quite useful for numerical implementations. As an example, we illustrate the application of our method to a celebrated free boundary problem. The results were announced in [11.
\end{abstract}

1. Introduction

\section{PLAN OF THE PAPER}

2. Setting of the primal problem

3. The duality principle

3.1. Heuristic genesis

3.2. The admissible fields

3.3. The dual problem

4. Convexification recipe

4.1. Construction of the convex extension of the primal energy

4.2. Integral representation of $H$

4.3. Generalized coarea formula

4.4. Proof of Theorem 4.1

5. Optimality conditions and min-max formulation

6. Application to a free boundary problem

6.1. Description of the problem

6.2. Numerical algorithms

6.3. Some simulations in case $N=1$

6.4. Some simulations in case $N=2$

7. Completion of the proofs

\section{INTRODUCTION}

A central issue of Convex Analysis is the development of a duality theory: this allows to associate with an initial convex variational problem a dual problem which has the same extremal value and in many cases is easier to solve; moreover, solutions to both primal and dual problem can be nicely characterized through necessary and sufficient optimality conditions. This is by now a very classical road, which in the last decades has found applications in different areas, such as mechanics, optimal control, economics, mass transportation, and many more. In fact, one of the reasons is that the duality approach enables to set up very stable and efficient approximation schemes. We refer the reader to

Date: June 30, 2021.

2010 Mathematics Subject Classification. 
the reference monograph [26] for the theoretical framework (see also [9]), and to [18, 32] for more recent surveys including applications and numerical algorithms.

Unfortunately, such theory completely breaks down as soon as some nonconvexity appears in the optimization problem under study. In particular, this drawback is often met in Calculus of Variations, where even very classical problems involve non-convex energy costs. As no systematical tool is available to characterize a global optimum, a dramatic consequence is that all currently available numerical methods loose their efficiency, because they are not able to rule out local minimizers and detect the global ones.

To have in mind a prototype situation, let us mention for instance the free boundary problem studied in the seminal paper [2]:

$$
\inf \left\{\int_{\Omega} \frac{1}{2}|\nabla u|^{2} d x+\lambda|\{u>0\}|: u \in H^{1}(\Omega), u=1 \text { on } \partial \Omega\right\},
$$

the free boundary being the frontier of the positivity set $\{u>0\}$ (see Figure 1). A huge literature about free boundaries stemmed from the existence and regularity results proved in 2] (without any attempt of completeness, see for instance [3, 22, 20, 21, 30, 33]). However, these papers are mainly focused on the study of local minimizers, through the Euler-Lagrange equation and the related free boundary condition, intended in the variational or in the viscosity sense. To the best of our knowledge, a systematic way to evidence global minimizers for problem (1.1) is still missing.

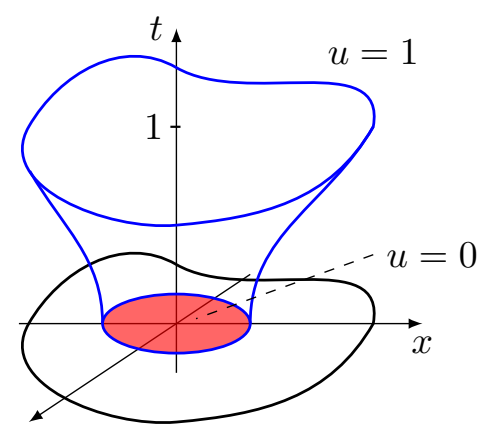

FiguRE 1. The free boundary problem 1.1

In this work we present a new duality theory for non-convex variational problems, which aims at filling the lack depicted so far. In this respect, the papers [1, 8, 28, 34, 35] should be mentioned among the few attempts outside the convex framework.

We consider very general minimization problems of the form

$$
\mathcal{I}:=\inf \left\{\int_{\Omega} f(u, \nabla u) d x+\int_{\Gamma_{1}} \gamma(u) d \mathcal{H}^{N-1}: u \in W^{1, p}(\Omega), u=u_{0} \text { on } \Gamma_{0}\right\},
$$

where $\Omega$ is an open bounded domain of $\mathbb{R}^{N}$ with a Lipschitz boundary and $\left(\Gamma_{0}, \Gamma_{1}\right)$ is a partition of $\partial \Omega$ : $\Gamma_{0}$ and $\Gamma_{1}$ correspond respectively to the Dirichlet part (the datum $u_{0}$ is a given function in $W^{1, p}(\Omega)$ ), and to the Neumann part of the boundary.

The bulk integrand $f: \mathbb{R} \times \mathbb{R}^{N} \rightarrow \mathbb{R}$ is assumed to lower semicontinuous in both variables, and convex in $z$, but the key point is that it may have a non-convex dependence in $t$.

The boundary integrand $\gamma$ is assumed to be Lipschitz, and suitable $p$-growth conditions are imposed on $f$ and $\gamma$ to ensure the existence of a minimizer in $W^{1, p}(\Omega)$ (for some $p>1$ ). 
Clearly, problem 1.1 falls into this general framework, by taking $\Gamma_{0}=\partial \Omega, u_{0} \equiv 1$, and $f(t, z)=\frac{1}{2}|z|^{2}+\chi_{(0,+\infty)}(t)$, where $\chi_{(0,+\infty)}$ is the characteristic function of $(0,+\infty)$.

As a further example, one can take $f(t, z):=\varepsilon|z|^{2}+W(t)-\lambda t, W$ being a two-wells potential, $\varepsilon$ a small positive parameter, and $\lambda$ a Lagrange multiplier. In this case, if $\Gamma_{1}=\partial \Omega$, problem $(1.2)$ describes the configuration of a Cahn-Hilliard fluid in presence of a wetting term $\gamma$ on the whole of the boundary.

For general minimization problems of the form $(1.2)$, the dual problem we propose is formulated as follows

$$
\mathcal{I}^{*}:=\sup \left\{\int_{G_{u_{0}}} \sigma \cdot \nu_{u_{0}} d \mathcal{H}^{N}+\int_{\Gamma_{1}} \gamma\left(u_{0}\right) d \mathcal{H}^{N-1}: \sigma \in \mathcal{B}\right\} .
$$

and any optimal $\sigma$ is called a calibration, in analogy to the case of classical principle of calibration for minimal surfaces (see [27, 31, 1] and references therein).

The class $\mathcal{B}$ of admissible competitors is a family of bounded divergence free vector fields $\sigma$, defined on $\Omega \times \mathbb{R}$, which have a given normal trace on $\Gamma_{1} \times \mathbb{R}$ and satisfy suitable convex pointwise constraints. The first integral appearing in (1.3) denotes the flux of $\sigma$ across the graph of the function $u_{0}$, and it is well-defined as admissible fields turn out to admit a normal trace on any set with finite perimeter. We refer to Section 3 for all the details, including the precise statement of the convex constraints satisfied by the admissible fields, and its comparison with the classical dual problem in the convex case.

Here let us just give the complete formulation in case of problem (1.1), when the dual problem reads:

$$
\mathcal{I}^{*}=\sup \left\{-\int_{\Omega} \sigma^{t}(x, 1) d x: \sigma \in \mathcal{B}\right\} .
$$

Notice that in this case the integral on $\Gamma_{1}$ is missing (since $\Gamma_{0}=\partial \Omega$ ), whereas the integral on $\Omega$ represents the flux term across the graph of the boundary datum $u_{0} \equiv 1$. Namely, $\sigma^{t}$ denotes the vertical component of an element $\sigma=\left(\sigma^{x}, \sigma^{t}\right)$ lying in the admissible class $\mathcal{B}$, which for the problem under consideration is given by all bounded divergence free vector field on $\Omega \times \mathbb{R}$ satisfying the constraints

$$
\sigma^{t}(x, t)+\lambda \geq \frac{1}{2}\left|\sigma^{x}(x, t)\right|^{2} \quad \text { a.e. on } \Omega \times \mathbb{R}, \quad \sigma^{t}(x, 0) \geq 0 \text { a.e. on } \Omega .
$$

Thus problem (1.4) has a nice fluid mechanic interpretation: it consists in maximizing the downflow through the top face $\Omega \times\{1\}$ of an incompressible fluid constrained into the cylinder $\Omega \times \mathbb{R}$, whose speed $\sigma$ satisfies the conditions above, preventing in particular the fluid to pass across the bottom face (see Figure 2 , in which $\Omega=(0,1)^{2} \subset \mathbb{R}^{2}$ ).

Our main result establishes that, in the general setting sketched above and fixed more precisely in Section 2, there is no duality gap: the infimum $\mathcal{I}$ in 1.2 and the supremum $\mathcal{I}^{*}$ in 1.3 coincide. The result is stated, along with several comments, in Section 3 (see Theorem 3.4 , after providing a heuristic description of the underlying idea, and giving all the required details about the class of admissible fields.

The proof is quite delicate and to it is devoted most part of the paper. Here we limit ourselves to give just few hints. The approach we adopt is based on the idea of reformulating the primal problem $(1.2)$ in $(N+1)$ space dimensions. More precisely, in the same spirit of what done in the paper [1] for the Mumford-Shah functional (see also [24]), the starting point is to identify any admissible function $u: \Omega \rightarrow \mathbb{R}$ with the characteristic function $\mathbb{1}_{u}$ of its subgraph. Then the building block of our method is a convexification recipe, which is carried over in Section 4. Roughly speaking, it consists in embedding the class $\mathcal{A}$ of 


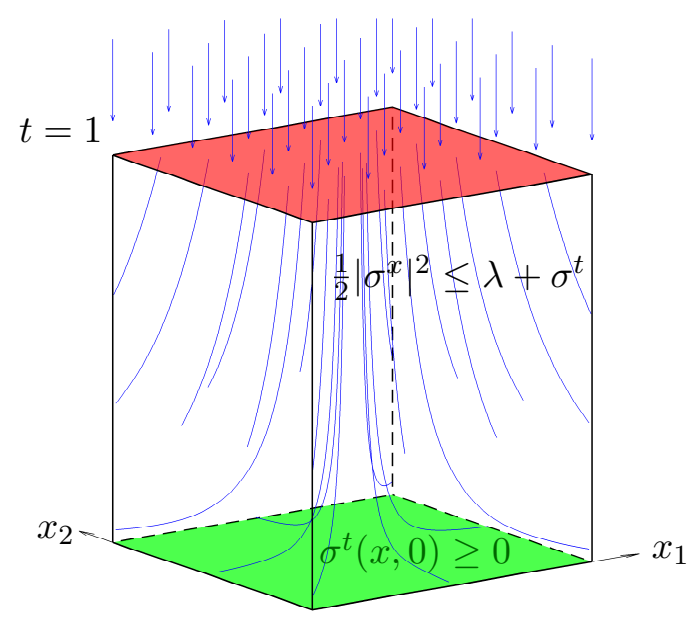

Figure 2. The optimal flow problem (1.4)

competitors for the primal problem $(1.2)$ into an enlarged class $\widehat{\mathcal{A}}$ of functions $v$ defined on $\Omega \times \mathbb{R}$ (via the identification $u \mapsto \mathbb{1}_{u}$ ), and in constructing a convex functional $\widehat{E}$, which extends the primal energy $E(u):=\int_{\Omega} f(u, \nabla u) d x+\int_{\Gamma_{1}} \gamma(u) d \mathcal{H}^{N-1}$ to the class $\widehat{\mathcal{A}}$. The key intermediate result (see Theorem 4.1) states that the infimum of the convex functional $\widehat{E}$ over the class $\widehat{\mathcal{A}}$ coincides with $\mathcal{I}$, and that the solutions to the two problems are closely related to each other. To establish such result, we exploit as a crucial ingredient a new very general coarea type formula (see Theorem 4.10).

The completion of the proof of Theorem 3.4 is postponed in Section 7 (since this last part is not needed for the comprehension of the contents of Sections 5 and 6). It is obtained essentially by using convex duality in $(N+1)$ space dimensions, in synergy with several ad-hoc arguments, driven from convex analysis and geometric measure theory, needed to handle the involved functions and fields.

The companion results of our duality theory are presented in Section 5 in Theorem 5.1 we show that solutions to the primal and to the dual problem can be characterized through an equality holding on the graph of an optimal function $\bar{u}$, and in Corollary 5.2 we give a practical way to check such condition in concrete situations; in Theorem 5.4 we reformulate our duality principle under the form of a min-max result, and a variant which is conceived especially for numerical purposes is added in Proposition 5.7.

In Section 6 we exemplify the application of our method to problem (1.1).

To conclude, let us stress that this paper aims to give a breakthrough by settling the bases of the non-convex duality theory, but of course it cannot contain the many developments which are expected and which will be studied in forthcoming works.

In particular, the existence of a solution to the dual problem, that we call a calibration, is a major issue. In the forthcoming paper [14, by using rearrangement techniques for integrals with non-constant densities, we are going to provide an existence result for problems with linear growth (for which a variant of Theorem 3.4 can be established). Moreover, the numerical results given in Section 6 will be detailed and expanded in [15]. 
As further open problems and possible generalizations, let us mention that our duality principle may be easily extended to the case when $f$ and $\gamma$ depend also on the spatial variable $x$. On the other hand, possible adaptations of the same idea to variational integrals involving the Hessian of $u$ are not straightforward and deserve further investigation. Finally, our results open the innovative perspective of studying the stability of minimizers of non-convex functionals by computing their shape derivatives (in fact, our duality result should allow to extend successfully to the non-convex setting the approach recently proposed in [12, 13]).

Acknowledgments. We acknowledge the financial support the University of Toulon, Politecnico di Milano, the University of Pavia, and the Italian institutions MIUR and INDAM, which helped the accomplishment of this work through PRIN and GNAMPA projects. We are very grateful to Cédric Galusinski and Minh Phan for handling the numerical simulations presented in Section 6 .

\section{Setting of the PRimal PROBlem}

Let $\Omega$ be an open bounded domain of $\mathbb{R}^{N}$, and let $\left(\Gamma_{0}, \Gamma_{1}\right)$ be a partition of $\partial \Omega$. We consider as primal problem the non-convex infimum problem

$$
\mathcal{I}:=\inf \{E(u): u \in \mathcal{A}\}
$$

where the energy cost is of the form

$$
E(u):=\int_{\Omega} f(u, \nabla u) d x+\int_{\Gamma_{1}} \gamma(u) d \mathcal{H}^{N-1},
$$

and the class of admissible functions is given by

$$
\mathcal{A}:=\left\{u \in W^{1, p}(\Omega): u=u_{0} \text { on } \Gamma_{0}\right\},
$$

being $u_{0}$ a fixed element in $W^{1, p}(\Omega)$.

We work under the setting of hypotheses listed hereafter.

\section{Standing assumptions:}

- The boundary $\partial \Omega$ is Lipschitz with unit outer normal $\nu_{\Omega}$.

- The integrand $f=f(t, z)$ is a function $f: \mathbb{R} \times \mathbb{R}^{N} \rightarrow(-\infty,+\infty]$ sastisfying:

$$
\begin{gathered}
\forall t \in \mathbb{R}, z \mapsto f(t, z) \text { is convex; } \\
(t, z) \mapsto f(t, z) \text { is lower semicontinuous on } \mathbb{R} \times \mathbb{R}^{N} ; \\
\forall(t, z) \in \mathbb{R} \times \mathbb{R}^{N}, f(t, z) \geq \alpha|z|^{p}-r(t),
\end{gathered}
$$

where $p \in(1,+\infty), \alpha$ is a positive constant, and $r=r(t)$ is a Borel function such that

$$
0 \leq r(t) \leq C \text { for some } C>0 \text {. }
$$

- There exists a Lebesgue negligible set of $D \subset \mathbb{R}$ such that, for every $z \in \mathbb{R}^{N}$, the map $t \mapsto f(t, z)$ is upper semicontinuous on $\mathbb{R} \backslash D$, namely

$$
f(t, z) \geq \limsup _{s \rightarrow t} f(s, z) \quad \forall z \in \mathbb{R}^{N}, \forall t \in \mathbb{R} \backslash D .
$$


- $\gamma: \mathbb{R} \rightarrow \mathbb{R}$ is a Lipschitz function such that $\gamma(0)=0$ and

$$
\begin{cases}\inf _{t \in \mathbb{R}} \gamma(t)>-\infty & \text { if } \Gamma_{0} \neq \emptyset \\ \liminf _{|t| \rightarrow+\infty} \frac{\gamma(t)}{|t|}>0 & \text { if } \Gamma_{0}=\emptyset,\end{cases}
$$

- The set $\{u \in \mathcal{A}: E(u)<+\infty\}$ is not empty.

Remark 2.1. (i) We emphasize that the function $f$ is not assumed to be convex in $t$.

(ii) We point out that, by taking $\Gamma_{1}=\partial \Omega$ and $\gamma=0$, we can handle homogeneous Neumann boundary conditions. Notice also that the condition $\gamma(0)=0$ is not restrictive up to adding a constant.

(iii) Allowing a nonempty discontinuity set $D$ of vanishing Lebesgue measure for the map $t \mapsto f(t, z)$ (according to (2.8)) is quite important in order to make our duality method applicable in case of free boundary problems, $c f$. Section 6 .

(iv) The boundedness of $r$ is a technical condition which will be exploited mainly in the proof of Lemma 7.1 .

(v) One of the main roles of the growth conditions (2.6) and (2.9) imposed respectively on $f$ and $\gamma$ is to ensure the well-posedness of the primal problem, as stated in the next result. (vi) We stress that, for the validity of Proposition 2.2 it is important to have chosen $p>1$ in (2.6), since for $p=1$ the primal problem may fail to admit a solution. The main reason is that in such case the energy $E$ is no longer lower semicontinuous (whereas coercivity still holds, as it is easy to see by inspection of the proof below). Thus one needs to relax the energy $E$ in $B V(\Omega)$ (see [25]), which is made extremely delicate by the presence of the boundary integral in (2.2), in particular when $\partial \Omega$ exhibits corners (see [16]).

On the other hand, with minor modifications in the proof, our duality Theorem 3.4 remains true also in the case $p=1$ (provided $\Gamma_{0}=\partial \Omega$ ), and this is precisely the setting in which it seems easier to obtain the existence of a solution for the dual problem. An existence result for the dual problem in the framework of nonconvex functionals with linear growth under Dirichlet boundary conditions will be the topic of a forthcoming paper.

Proposition 2.2. (well-posedness of the primal problem) The infimum $\mathcal{I}$ in (2.1) is finite and attained.

Proof. Since we assumed that the class $\mathcal{A}$ of admissible competitors contains some element $u$ of finite energy, we may apply the direct method of the Calculus of Variations. Thus we are reduced to showing that, under the standing assumptions, the energy $E$ defined in 2.2 is both lower semicontinuous and coercive respect to the weak topology of $W^{1, p}(\Omega)$. The weak lower semicontinuity of the first addendum of the functional $E$ follows wellknown results of weak-strong convergence (see for instance [19, Chapter 4]), which can be applied in particular thanks to the growth conditions (2.6).

The weak lower semicontinuity of the second addendum follows as a consequence of the compact embedding of $W^{1, p}(\Omega)$ into $L^{p}(\partial \Omega)$, by applying Fatou's lemma.

We then focus attention on the coercivity property. We claim that there exists positive constants $C_{1}, C_{2}$ such that

$$
E(u) \geq C_{1}\|u\|_{W^{1, p}(\Omega)}-C_{2} .
$$

In case $\Gamma_{1}=\emptyset$, the coercivity follows immediately from the lower bound in $(2.6)$, taking into account that $r(t)$ satisfies 2.7. 


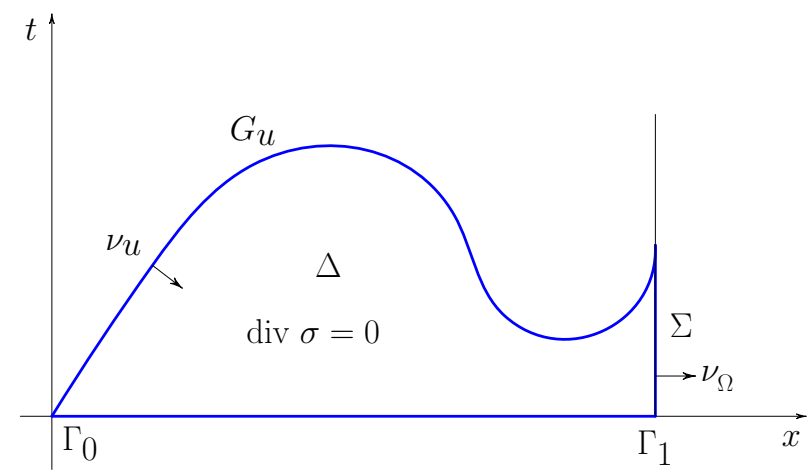

In case $\Gamma_{1} \neq \emptyset$, we further distinguish the cases $\Gamma_{0} \neq \emptyset$ and $\Gamma_{0}=\emptyset$. If $\Gamma_{0} \neq \emptyset$, the coercivity follows again from the lower bound in (2.6), taking into account that $r(t)$ satisfies $(2.7)$. If $\Gamma_{0}=\emptyset$, the lower bound in $(2.6)$ tells us merely that $u_{n}$ are bounded in $W^{1, p}(\Omega)$ modulo constants, but by invoking the second condition in (2.9), we obtain that the boundary traces of $u_{n}$ are bounded in $L^{1}(\partial \Omega)$, and hence the constants are bounded.

\section{The DuAlity PRinciple}

In this section we present our new duality principle:

- in Subsection 3.1 we provide an intuitive presentation of the underlying idea;

- in Subsection 3.2 we introduce the class of admissible fields in the dual problem;

- in Subsection 3.3 we state the result (see Theorem 3.4), along with some basic remarks.

3.1. Heuristic genesis. The original idea, already exploited in [1 for free-discontinuity problems, relies on geometric measure theory and stems from the so-called calibration method for minimal surfaces (see [31, 27]). It consists in considering a suitable convex set $\mathcal{K}$ of vector fields $\sigma=\left(\sigma^{x}, \sigma^{t}\right): \Omega \times \mathbb{R} \rightarrow \mathbb{R}^{N+1}$ satisfying the following requirement:

$$
\int_{\Omega} f(u, \nabla u) d x=\sup _{\sigma \in \mathcal{K}} \int_{G_{u}} \sigma \cdot \nu_{u} d \mathcal{H}^{N} \quad \forall u \in W^{1, p}(\Omega) .
$$

The integral at the right hand side of (3.1), which is well defined provided $\sigma$ is regular enough, represents the flux of $\sigma$ across the graph $G_{u}$ of $u$, seen as a $N$-dimensional rectifiable subset of $\mathbb{R}^{N+1}$ and endowed with oriented unit normal

$$
\nu_{u}=\frac{(\nabla u,-1)}{\sqrt{1+|\nabla u|^{2}}} .
$$

Given a function $u$ in $W^{1, p}(\Omega)$ such that $u=u_{0}$ on $\Gamma_{0}$, we denote by $\Delta$ the subset of $\Omega \times \mathbb{R}$ lying between $G_{u_{0}}$ and $G_{u}$, and by $\Sigma \subset \Gamma_{1} \times \mathbb{R}$ the "lateral part" of $\partial \Delta$, namely the set of points $(x, t)$ with $x \in \Gamma_{1}$, and $t$ between $u_{0}(x)$ and $u(x)$. In case $N=1$, taking $u_{0}=0$ and $u \geq 0$, the region $\Delta$ is represented in Figure 3.1 .

Let now $\sigma$ be a smooth element belonging to a class $\mathcal{K}$ verifying (3.1), and assume that $\sigma$ satisfies the additional conditions

$$
\operatorname{div} \sigma=0 \text { in } \Omega \times \mathbb{R} \quad \text { and } \quad \sigma \cdot \nu_{\Omega}=-\gamma^{\prime} \text { on } \Gamma_{1} \times \mathbb{R} .
$$


By applying the divergence theorem on the region $\Delta$, we obtain:

$$
\begin{aligned}
\int_{G_{u}} \sigma \cdot \nu_{u} d \mathcal{H}^{N}-\int_{G_{u_{0}}} \sigma \cdot \nu_{u_{0}} d \mathcal{H}^{N} & =\int_{\Sigma} \operatorname{sign}\left(u-u_{0}\right) \sigma \cdot \nu_{\Omega} d \mathcal{H}^{N} \\
& =-\int_{\Sigma} \operatorname{sign}\left(u(x)-u_{0}(x)\right) \gamma^{\prime}(t) d \mathcal{H}^{N-1}(x) d t \\
& =\int_{\Gamma_{1}}\left(\gamma\left(u_{0}\right)-\gamma(u)\right) d \mathcal{H}^{N-1}
\end{aligned}
$$

In view of (3.1), and recalling the definition 2.2 of the energy $E$, we deduce that

$$
E(u) \geq \int_{G_{u_{0}}} \sigma \cdot \nu_{u_{0}} d \mathcal{H}^{N}+\int_{\Gamma_{1}} \gamma\left(u_{0}\right) d \mathcal{H}^{N-1} .
$$

It is then natural to optimize the above inequality by considering the linear programming problem

$$
\sup \left\{\int_{G_{u_{0}}} \sigma \cdot \nu_{u_{0}} d \mathcal{H}^{N}+\int_{\Gamma_{1}} \gamma\left(u_{0}\right) d \mathcal{H}^{N-1}: \sigma \in \mathcal{K} \text { satisfying } 3.3 p\right\} .
$$

Clearly from the above discussion the supremum in (3.4) turns out to be bounded from above by the infimum $\mathcal{I}$ of the primal problem. We have thus found a linear programming problem which is a good candidate for being the dual problem. To elect it as such, we have to complete the plan, by choosing $\mathcal{K}$ so that the equality (3.1) holds and the supremum in (3.4) equals $\mathcal{I}$.

Let us now focus our attention on the construction of the class $\mathcal{K}$, by giving some heuristic arguments (the rigorous definition is postponed to Section 3.2 below).

Assume that $\sigma=\left(\sigma^{x}, \sigma^{t}\right) \in \mathcal{C}^{1}\left(\Omega \times \mathbb{R} ; \mathbb{R}^{N+1}\right)$ satisfies the pointwise inequality

$$
\sigma^{t}(x, t) \geq f_{z}^{*}\left(t, \sigma^{x}(x, t)\right) \quad \forall(x, t) \in \Omega \times \mathbb{R},
$$

where $f_{z}^{*}$ denotes the Fenchel conjugate of $f$ with respect to $z$ :

$$
f_{z}^{*}\left(t, z^{*}\right):=\sup _{z \in \mathbb{R}^{N}}\left[z \cdot z^{*}-f(t, z)\right] .
$$

By using (3.5) on the graph of $u$ and the Fenchel inequality, we obtain

$$
\begin{aligned}
\int_{\Omega} f(u, \nabla u) d x & \geq \int_{\Omega}\left[f_{z}^{*}\left(u(x), \sigma^{x}(x, u(x))\right)+f(x, \nabla u(x))-\sigma^{t}(x, u(x))\right] d x \\
& \geq \int_{\Omega}\left[\sigma^{x}(x, u(x)) \cdot \nabla u-\sigma^{t}(x, u(x))\right] d x=\int_{G_{u}} \sigma \cdot \nu_{u} d \mathcal{H}^{N} .
\end{aligned}
$$

The above inequality turns out to optimal: actually, as it will be shown later, if $\mathcal{K}$ is chosen as the class of fields in $\mathcal{C}^{1}\left(\Omega \times \mathbb{R} ; \mathbb{R}^{N+1}\right)$ satisfying (3.5), not only the equality (3.1) holds true, but in addition the supremum in (3.4) equals $\mathcal{I}$.

However, the class of competitors we are going to choose in our dual problem has also to be large enough in order to allow the existence of optimal fields. In this respect, it will be clear from the examples considered in Section 6 that one cannot expect optimal fields to be $\mathcal{C}^{1}$ regular, and not even to be continuous fields which satisfy the inequality (3.5) pointwise at every $(x, t)$ in $\Omega \times \mathbb{R}$.

We are thus led to relax condition (3.5) and to work with fields which are less regular, but still admit a mathematically meaninfgul notion of flux and normal trace. 
3.2. The admissible fields. We consider the space

$$
X_{1}(\Omega \times \mathbb{R}):=\left\{\sigma \in L^{\infty}\left(\Omega \times \mathbb{R} ; \mathbb{R}^{N+1}\right): \operatorname{div} \sigma \in L^{1}(\Omega \times \mathbb{R})\right\},
$$

where the divergence is intended in distributional sense.

For any $\sigma \in X_{1}(\Omega \times \mathbb{R})$, a notion of weak normal trace can be defined as follows. Given an open set $A \subset \Omega \times \mathbb{R}$ with Lipschitz boundary and unit outer normal $\nu_{A}$, there exists a unique function $\sigma \cdot \nu_{A} \in L^{\infty}(\partial \Omega)$ such that

$$
\int_{\partial A}\left(\sigma \cdot \nu_{A}\right) \varphi d \mathcal{H}^{N}=\int_{A}(\sigma \cdot \nabla \varphi+\varphi \operatorname{div} \sigma) d x \quad \forall \varphi \in \mathcal{C}_{0}^{\infty}(\Omega \times \mathbb{R}) .
$$

The same assertion remains true when $A$ is merely a Lebesgue measurable set with finite perimeter, provided $\partial A$ is intended as the reduced boundary of $A$, and $\nu_{A}$ as the measure theoretic unit normal vector defined $\mathcal{H}^{N}$-a.e. on $\partial A$.

In particular, for any field $\sigma \in X_{1}(\Omega \times \mathbb{R})$ and any function $u \in W^{1, p}(\Omega)$, the flux integral

$$
\int_{G_{u}} \sigma \cdot \nu_{u} d \mathcal{H}^{N}
$$

is well-defined according to (3.7) (precisely, by taking as a set $A$ the subgraph of $u$, we have $\nu_{A}=-\nu_{u}$, with $\nu_{u}$ given by (3.2)).

For later use, let us notice that, as (3.7) can be extended to all $\varphi \in L^{\infty}(A) \cap W^{1,1}(A)$, a duality argument easily yields the following equality

$$
\begin{aligned}
& \left\{\left(-\operatorname{div} q, q \cdot \nu_{A}\right): q \in X_{1}(A)\right\}= \\
& \left\{(f, g) \in L^{1}(A) \times L^{\infty}(\partial A): \int_{A} f d x+\int_{\partial A} g d \mathcal{H}^{N}=0\right\} .
\end{aligned}
$$

We refer to [6, 20] for more details on these topics (see also Section 4.5, where we shall need to exploit a generalized version of the Gauss-Green Theorem involving BV functions).

Definition 3.1. (i) We set $\mathcal{K}$ the class of fields $\sigma=\left(\sigma^{x}, \sigma^{t}\right) \in X_{1}(\Omega \times \mathbb{R})$ such that

$$
\begin{gathered}
\sigma^{t}(x, t) \geq f_{z}^{*}\left(t, \sigma^{x}(x, t)\right) \text { for } \mathcal{L}^{N+1} \text {-a.e. }(x, t) \in \Omega \times \mathbb{R} \\
\sigma^{t}(x, t) \geq-f(t, 0) \quad \forall t \in D \text { and for } \mathcal{L}^{N} \text {-a.e. } x \in \Omega,
\end{gathered}
$$

where $D$ is the Lebesgue negligible set introduced in the standing assumption (2.8).

(ii) We denote by $\mathcal{B}$ the class of fields $\sigma \in \mathcal{K}$ satisfying the following two conditions:

$$
\begin{gathered}
\operatorname{div} \sigma=0 \text { in } \Omega \times \mathbb{R} \\
\sigma^{x} \cdot \nu_{\Omega}=-\gamma^{\prime} \text { on } \Gamma_{1} \times \mathbb{R},
\end{gathered}
$$

where $\sigma^{x} \cdot \nu_{\Omega}$ is meant as the weak normal trace of $\sigma$ on $\partial(\Omega \times \mathbb{R})\left(\right.$ as $\left.\nu_{\Omega \times \mathbb{R}}=\left(\nu_{\Omega}, 0\right)\right)$.

Remark 3.2. Few comments are in order about condition (3.10), which did not appear in our previous heuristic discussion. First we observe that, for every fixed $t \in D, \sigma^{t}(\cdot, t)$ makes sense as the weak normal trace of $\sigma$ on $\Omega \times\{t\}$ according to (3.7). The role of condition (3.10) is to make the class of admissible fields sensitive to the possible discontinuities of the integrand $f$. In this respect, the almost everywhere inequality $(3.9)$ alone would be too weak, since it is independent from the behaviour of $f$ on sets of vanishing measure: for instance, condition (3.9) reads exactly the same in the two cases when $f(t, z)=\frac{1}{2} z^{2}+\chi_{\{t \neq 0\}}$ or $f(t, z)=\frac{1}{2} z^{2}+1$ (since the discontinuity set $\{t=0\}$ is $\mathcal{L}^{N+1}$-negligible). Finally, let us mention that the inequality (3.10) is actually satisfied also on the complement of $D$. 
Namely we shall see later on that, for any $\sigma \in \mathcal{B}$, there holds $\sigma^{t}(x, t) \geq f(t, 0)$ for every $t \in \mathbb{R}$ and $\mathcal{L}^{N}$-a.e. $x \in \Omega(c f$. Remark 4.7).

Remark 3.3. Conditions 3.9 -3.10 can be rephrased as

$$
\begin{gathered}
\sigma(x, t) \in K(t) \quad \text { for } \mathcal{L}^{N+1} \text {-a.e. }(x, t) \in \Omega \times \mathbb{R}, \\
\sigma^{t}(x, t) \in \Pi_{N+1}[K(t)] \quad \forall t \in D \text { and for } \mathcal{L}^{N} \text {-a.e. } x \in \Omega,
\end{gathered}
$$

where $K(t)$ is the convex subset of $\mathbb{R}^{N+1}$ given for every $t \in \mathbb{R}$ by

$$
K(t):=\left\{q=\left(q^{x}, q^{t}\right): q^{t} \geq f_{z}^{*}\left(t, q^{x}\right)\right\},
$$

and $\Pi_{N+1}[\cdot]$ denotes the projection on the last component of $\mathbb{R}^{N+1}$ (that is, on the space spanned by $e_{N+1}=(0,1)$ ). In particular, the equivalence between 3.10) and (3.14) follows from the identity $\inf _{z^{*}} f_{z}^{*}\left(t, z^{*}\right)=-f(t, 0)$.

3.3. The dual problem. Recall that $u_{0} \in W^{1, p}(\Omega)$ is the prescribed trace on the Dirichlet part $\Gamma_{0}$ of the boundary $\left(c f .(2.3)\right.$ ), that $\gamma$ is the energy density on the Neumann part $\Gamma_{1}$ of the boundary $(c f .(2.2)$ ), and that, for every field $\sigma$ belonging to the class $\mathcal{B}$ introduced in Definition 3.1, the flux across the graph of $u_{0}$ is well-defined as explained in Section 3.2 . We set

$$
\left(\mathcal{P}^{*}\right)
$$

$$
\mathcal{I}^{*}:=\sup \left\{\int_{G_{u_{0}}} \sigma \cdot \nu_{u_{0}} d \mathcal{H}^{N}+\int_{\Gamma_{1}} \gamma\left(u_{0}\right) d \mathcal{H}^{N-1}: \sigma \in \mathcal{B}\right\}
$$

The core of our duality theory is the following

Theorem 3.4 (duality principle). The extrema of the primal and dual problems defined respectively in (2.1) and (3.16) coincide:

$$
\mathcal{I}=\mathcal{I}^{*} .
$$

Several comments are listed in the next remarks.

Remark 3.5. In the pure Neumann case when $\Gamma_{0}=\emptyset$ (so that the boundary datum $u_{0}$ is not defined), definition (3.16) must be intended as if $u_{0}=0$, namely $\mathcal{I}^{*}$ can be reformulated as $(c f .[11])$

$$
\mathcal{I}^{*}=\sup \left\{-\int_{\Omega} \sigma^{t}(x, 0) d x: \sigma \in \mathcal{B}\right\} .
$$

Remark 3.6. In many cases, when the boundary datum $u_{0}$ is a bounded function, there exist a priori lower or upper bounds for the minimizers of the primal problem $(\mathcal{P})$, so that the infimum value $\mathcal{I}$ is unchanged if we impose $u$ to take values in a suitable closed interval $[m, M]$ of the real line. We are thus led to consider the variant of the primal problem (2.1) where the class of admissible functions is changed into

$$
\mathcal{A}(m, M):=\left\{u \in W^{1, p}(\Omega ;[m, M]): u=u_{0} \text { on } \Gamma_{0}\right\}
$$

In this case, our duality result continues to hold (with a simpler proof, see Proposition 5.7), provided the admissible fields in the dual problem $\left(\mathcal{P}^{*}\right)$ are taken in the class $\mathcal{B}(m, M)$ of 
elements $\sigma \in X_{1}(\Omega \times(m, M))$ satisfying:

$$
\begin{gathered}
\sigma^{t}(x, t) \geq f_{z}^{*}\left(t, \sigma^{x}(x, t)\right) \text { for } \mathcal{L}^{N+1} \text {-a.e. }(x, t) \in \Omega \times(m, M) \\
\sigma^{t}(x, t) \geq-f(t, 0) \quad \forall t \in D \cup\{m, M\} \text { and for } \mathcal{L}^{N} \text {-a.e. } x \in \Omega \\
\operatorname{div} \sigma=0 \text { in } \Omega \times(m, M) \\
\sigma^{x} \cdot \nu_{\Omega}=-\gamma^{\prime} \text { on } \Gamma_{1} \times(m, M) .
\end{gathered}
$$

This reduction of the dual problem to a bounded set will be of course crucial in the implementation of efficient algorithms for the numerical approximation of its solutions.

Remark 3.7. In general the solution to the dual problem $\left(\mathcal{P}^{*}\right)$ is not unique (see Section 6). However, if the infimum of $(\mathcal{P})$ is reached in $\mathcal{A}(m, M)$ and the supremum of $\left(\mathcal{P}^{*}\right)$ is reached in $\mathcal{B}(m, M)$, then a unique solution to $\left(\mathcal{P}^{*}\right)$ can be selected by considering the Tikhonov regularization

$$
\sup \left\{\int_{G_{u_{0}}} \sigma \cdot \nu_{u_{0}} d \mathcal{H}^{N}+\int_{\Gamma_{1}} \gamma\left(u_{0}\right)-\varepsilon \int_{\Omega \times(m, M)}|\sigma|^{2} d x: \sigma \in \mathcal{B}(m, M)\right\} .
$$

As $\varepsilon \rightarrow 0$, we are led to the solution of minimal $L^{2}$-norm.

Remark 3.8. In case the integrand $f$ is convex in $(t, z)$, the inequality $\mathcal{I}^{*} \geq \mathcal{I}$ (which is the most delicate part in the proof of Theorem 3.4 is a straightforward consequence of classical duality theory. To see this, consider vector fields of the form $\sigma(x, t)=(\eta(x), a(x)-$ $t \operatorname{div} \eta(x))$. For such fields, the inequality $\sigma^{t}(x, t) \geq f_{z}^{*}\left(t, \sigma^{x}(x, t)\right)$ is satisfied if and only if

$$
a(x) \geq \sup _{t}\left\{t \operatorname{div} \eta(x)+f_{z}^{*}(t, \eta)\right\}=\sup _{(z, t)}\{t \operatorname{div} \eta+z \cdot \eta-f(t, z)\}=f^{*}(\operatorname{div} \eta, \eta) .
$$

We deduce that $\mathcal{B}$ contains the class $\Theta$ given by fields of the form $\sigma(x, t)=(\eta(x), a(x)-$ $t \operatorname{div} \eta(x))$, with $\eta \in \mathcal{C}^{1}\left(\bar{\Omega} ; \mathbb{R}^{N}\right), \eta \cdot \nu_{\Omega}=-\gamma^{\prime}$ on $\Gamma_{1}$, and $a \in \mathcal{C}^{0}(\Omega), a(x) \geq f^{*}(\operatorname{div} \eta, \eta)$ in $\Omega \times \mathbb{R}$. Therefore,

$$
\begin{aligned}
\mathcal{I}^{*} & \geq \sup \left\{\int_{\Omega}-\sigma^{t}(x, 0) d x: \sigma \in \Theta\right\} \\
& =\sup \left\{\int_{\Omega}-f^{*}(\operatorname{div} \eta, \eta) d x: \eta \in \mathcal{C}^{1}\left(\bar{\Omega} ; \mathbb{R}^{N}\right), \eta \cdot \nu_{\Omega}=-\gamma^{\prime} \text { on } \Gamma_{1}\right\} .
\end{aligned}
$$

The variational problem in the last line is the classical dual problem of $(\mathcal{P})$, and its supremum coincides with $\mathcal{I}$ by standard convex duality (see for instance [9, 26]).

Remark 3.9. In case $N=1$, when the variational problem $(\mathcal{P})$ is settled on an interval $(0, h)$ of the real line, every competitor $\sigma$ in the dual problem is a bounded divergence free vector field on $(0, h) \times \mathbb{R}$, so that it can been written under the form $\sigma=\left(\partial_{t} w,-\partial_{x} w\right)$, for some function $w \in \operatorname{Lip}((0, h) \times \mathbb{R})$. For instance, in the pure Dirichlet case $\Gamma_{0}=\{0, h\}$ with boundary conditions $u(0)=u(h)=c$, when the primal problem reads

$$
\mathcal{I}=\inf \left\{\int_{0}^{h} f\left(u, u^{\prime}\right) d t: u \in H^{1}(0, h), u(0)=u(h)=c\right\}
$$

the dual problem (3.16) written in terms of rotated gradients becomes:

$$
\begin{aligned}
\mathcal{I}^{*}=\sup \{w(h, c)-w(0, c): \quad & w \in \operatorname{Lip}((0, h) \times \mathbb{R}), \\
& -\partial_{x} w \geq f_{z}^{*}\left(t, \partial_{t} w\right) \mathcal{L}^{2} \text {-a.e. on }(0, h) \times \mathbb{R}, \\
& \left.-\partial_{x} w \geq-f(t, 0) \forall t \in D, \mathcal{L}^{1} \text {-a.e. on }(0, h)\right\} .
\end{aligned}
$$


Notice that problem (3.25) looks like the dual formulation of Monge-Kantorowich transport problem, with marginals equal to the Dirac masses at $(0, c)$ and $(h, c)$, and a modified gradient constraint with respect to the usual one $|\nabla w| \leq 1$.

Inspired by dynamic programming and optimal control, a natural candidate to solve 3.25 is the value function

$$
V(x, t):=\inf \left\{\int_{0}^{x} f\left(u, u^{\prime}\right) d s: u \in H^{1}(0, h), u(0)=c, u(x)=t\right\},
$$

or equivalently a candidate calibration is the rotated gradient $\left(-\partial_{t} V, \partial_{x} V\right)$.

Indeed, if $V$ is admissible in (3.25), it is automatically optimal. Namely, $\mathcal{I}^{*} \geq V(h, c)-$ $V(0, c)=\mathcal{I}$ and by Theorem 3.4 the first inequality holds necessarily as an equality.

Thus the key point is to check the admissibility of $V$ in problem (3.25). By using Bellman's optimality principle (see for instance [23, Theorem 1.2.2]), it is easy to check that $V$ satisfies the constraints asked in (3.25) at every differentiability point. Unfortunately, it misses to satisfy the last important requirement of being Lipschitz regular close to $s=0$. In Section 6 we shall be back to this phenomenon in connection with a relevant example of free boundary problem.

\section{Convexification ReCipe}

The synopsis of this section is the following:

- in Subection 4.1 we introduce a convex functional $\widehat{E}$, defined in one more space dimension, of the form $H+\ell$, with $H$ and $\ell$ conceived respectively with the aim of extending the bulk and the surface parts of the primal non-convex energy $E$; then we state the main result of the section (Theorem 4.1), which makes the link between the primal problem (2.1) and a minimization problem for $\widehat{E}$.

- in Subsection 4.2 we provide an integral representation result for $H$;

- in Subsection 4.3 we state a generalized coarea formula, which turns out to be satisfied in particular by $H$ (as it can be seen thanks to its integral representation);

- in Subsection 4.4 we prove Theorem 4.1 (by using in particular a slicing formula for $\widehat{E}$ which follows from the coarea formula for $H$ );

- in Subsection 4.5 we prove the inequality $\mathcal{I} \geq \mathcal{I}^{*}$, which is the easiest half of Theorem 3.4.

4.1. Construction of the convex extension of the primal energy. As enlightened by the heuristics given in Section 3.1, the basic idea of our duality method is to consider the flux of suitable fields across the graph of functions $u$ admissible in the primal problem; and, along this way, we are naturally led to apply the divergence theorem on subgraphs.

Let us now fix these ideas in a systematic setting, and develop them into the proposal of a convexification recipe: it consists in extending the non-convex energy introduced in 2.2 . to a convex functional defined in one more space dimension.

Any element $u$ of $H^{1}(\Omega)$ can be identified with a function in one more dimension, given by the characteristic function $\mathbb{1}_{u}$ of its subgraph, defined on $\Omega \times \mathbb{R}$ by

$$
\mathbb{1}_{u}(x, t):= \begin{cases}1 & \text { if } t \leq u(x) \\ 0 & \text { if } t>u(x)\end{cases}
$$


Notice that $\mathbb{1}_{u}$ is not in $L^{1}(\Omega \times \mathbb{R})$, but merely in $L_{\text {loc }}^{1}(\Omega \times \mathbb{R})$.

Our target is to find a convex lower semicontinuous functional $\widehat{E}: L_{\text {loc }}^{1}(\Omega \times \mathbb{R}) \rightarrow \mathbb{R} \cup\{+\infty\}$ and a suitable subclass $\widehat{\mathcal{A}}$ of $L_{\text {loc }}^{1}(\Omega \times \mathbb{R})$ such that:

- for every $u \in \mathcal{A}$, it holds $\mathbb{1}_{u} \in \widehat{\mathcal{A}}$ and $\widehat{E}\left(\mathbb{1}_{u}\right)=E(u)$;

- the infimum $\mathcal{I}$ in $(2.1)$ can be recast by minimizing $\widehat{E}$ over the class $\widehat{\mathcal{A}}$.

To that aim we are going to consider separately the bulk part and the surface part of the energy $E$.

We start by recalling that, for any $u \in W^{1, p}(\Omega)$ (and actually more in general for any $u \in$ $B V(\Omega)$ ), its subgraph is a set with finite perimeter [29, p. 371], or equivalently $D \mathbb{1}_{u}$ belongs to the space $\mathcal{M}\left(\Omega \times \mathbb{R} ; \mathbb{R}^{N+1}\right)$ of vector valued bounded measures on $\Omega \times \mathbb{R}$. However, $\mathbb{1}_{u}$ does not belong to $B V(\Omega \times \mathbb{R})$, since as already noticed it is not in $L^{1}(\Omega \times \mathbb{R})$, but merely bounded. We can thus say that $\mathbb{1}_{u}$ belongs to the following subspace of $L_{\text {loc }}^{1}(\Omega \times \mathbb{R})$ :

$$
B V_{\infty}(\Omega \times \mathbb{R}):=\left\{v \in L^{\infty}(\Omega \times \mathbb{R}): D v \in \mathcal{M}\left(\Omega \times \mathbb{R} ; \mathbb{R}^{N+1}\right)\right\} .
$$

For any $v \in B V_{\infty}(\Omega \times \mathbb{R})$ and any $\sigma$ in the space $X_{1}(\Omega \times \mathbb{R})$ defined in (3.6), a pairing $\sigma \cdot D v$ can be defined as the following linear functional, which turns out to be a Radon measure on $\Omega \times \mathbb{R}$ (see [6. Thm 1.5 and Corollary 1.6])

$$
\langle(\sigma \cdot D v), \varphi\rangle:=-\int_{\Omega \times \mathbb{R}} v(\sigma \cdot \nabla \varphi+\varphi \operatorname{div} \sigma) d x \quad \forall \varphi \in \mathcal{C}_{0}^{\infty}(\Omega \times \mathbb{R}) .
$$

Moreover this measure is absolutely continuous with respect to $|D v|$ and satisfies

$$
\int_{\Omega \times \mathbb{R}}|(\sigma \cdot D v)| \leq\|\sigma\|_{\infty} \int_{\Omega \times \mathbb{R}}|D v| .
$$

Notice that definition 4.2 reduces to 3.7 in the special case when $v$ is the characteristic function of a set $A \subset \Omega \times \mathbb{R}$ with finite perimeter.

We are now in a position to define on $L_{\text {loc }}^{1}(\Omega \times \mathbb{R})$ the following functional, which will give the required convex extension of the bulk part of the energy $E$ :

$$
H(v):= \begin{cases}\sup \left\{\int_{\Omega \times \mathbb{R}} \sigma \cdot D v: \sigma \in \mathcal{K}\right\} & \text { if } v \in B V_{\infty}(\Omega \times \mathbb{R}) \\ +\infty & \text { otherwise. }\end{cases}
$$

An integral representation result for $H$ will be proved in Subsection 4.2 below. In particular, such result will disclose the crucial information that any function $v \in L_{\text {loc }}^{1}(\Omega \times \mathbb{R})$ lying in the finiteness domain of $H$ satisfies a monotonicity condition, namely:

$$
H(v)<+\infty \Rightarrow \text { for } \mathcal{L}^{N} \text {-a.e. } x \in \Omega \text {, the map } t \mapsto v(x, t) \text { is decreasing. }
$$

We infer that, if $H(v)<+\infty$, for $\mathcal{L}^{N}$-a.e. $x \in \Omega$ and $\mathcal{L}^{1}$-a.e. $s \in(0,1)$, the set $\{\tau \in \mathbb{R}$ : $v(x, \tau) \leq s\}$ is a nonempty half-line, and we can define for later use the function

$$
u_{s}(x):=\inf \{\tau \in \mathbb{R}: v(x, \tau) \leq s\} .
$$

Notice that by construction the subgraph of $u_{s}$ agrees up to a Lebesgue negligible set with the level set $\{\tau \in \mathbb{R}: v(x, \tau)>s\}$, namely

$$
\mathbb{1}_{u_{s}}(x, t)=\chi_{\{v>s\}}(x, t) \quad \text { for } \mathcal{L}^{N+1} \text {-a.e. }(x, t) \in \Omega \times \mathbb{R} .
$$

Next we turn our attention to extend also the surface part of the energy $E$. To that aim we observe that, though $\mathbb{1}_{u} \notin L^{1}(\Omega \times \mathbb{R})$, it becomes integrable after a suitable translation. 
Indeed, since $u$ is almost everywhere finite, for a.e. $x \in \Omega$ the map $t \mapsto \mathbb{1}_{u}(x, t)$ is monotone decreasing, with

$$
\mathbb{1}_{u}(x,-\infty)=1 \quad \text { and } \quad \mathbb{1}_{u}(x,+\infty)=0 .
$$

We are thus led to introduce the reference function

$$
v_{0}(x, t):= \begin{cases}1 & \text { if } t \leq 0 \\ 0 & \text { if } t>0 .\end{cases}
$$

The equality

$$
\int_{\mathbb{R}}\left|\mathbb{1}_{u}(x, t)-v_{0}(x, t)\right| d t=|u(x)|,
$$

implies that $\mathbb{1}_{u}-v_{0} \in L^{1}(\Omega \times \mathbb{R})$ as soon as $u \in L^{1}(\Omega)$.

We infer that the class $\mathcal{A}$ introduced in $(2.3)$ can be embedded, through the map $u \mapsto \mathbb{1}_{u}$, into the class

$$
\widehat{\mathcal{A}}:=\left\{v \in B V_{\infty}(\Omega \times \mathbb{R}): v-v_{0} \in L^{1}(\Omega \times \mathbb{R}), v-\mathbb{1}_{u_{0}}=0 \text { on } \Gamma_{0} \times \mathbb{R}\right\},
$$

where the last equality is intended in the sense of traces.

Notice in particular that, for every $v \in \widehat{\mathcal{A}}$, the function $v-v_{0}$ is in $B V(\Omega \times \mathbb{R})$, so that it has a $L^{1}$-trace on $\Gamma_{1} \times \mathbb{R}$.

We are then in a position to define on $L_{\text {loc }}^{1}(\Omega \times \mathbb{R})$ the following functional, which will give the required convex extension of the surface part of the energy $E$ :

$$
\ell(v):= \begin{cases}\int_{\Gamma_{1} \times \mathbb{R}} \gamma^{\prime}(t)\left(v-v_{0}\right) d \mathcal{H}^{N-1} d t & \text { if } v \in \widehat{\mathcal{A}} \\ +\infty & \text { otherwise. }\end{cases}
$$

Finally, we set

$$
\widehat{E}(v):=H(v)+\ell(v) \quad \forall v \in L_{\mathrm{loc}}^{1}(\Omega \times \mathbb{R}) .
$$

The next result states that the functional $\widehat{E}$ and the class $\widehat{\mathcal{A}}$ thus defined fit exactly the target conditions demanded at the beginning of this section:

Theorem 4.1. (link between the initial non-convex problem and its convex extension) There holds

$$
\begin{aligned}
& \widehat{E}\left(\mathbb{1}_{u}\right)= \begin{cases}E(u) & \text { if } u \in W^{1, p}(\Omega) \\
+\infty & \text { if } u \in B V(\Omega) \backslash W^{1, p}(\Omega)\end{cases} \\
& \inf \{E(u): u \in \mathcal{A}\}=\inf \{\widehat{E}(v): v \in \widehat{\mathcal{A}}\} .
\end{aligned}
$$

Moreover, both the infima in 4.13 are finite and attained, and:

- if $u \in \operatorname{argmin}_{\mathcal{A}}(E)$, then $\mathbb{1}_{u} \in \operatorname{argmin}_{\widehat{\mathcal{A}}}(\widehat{E})$;

- if $v \in \operatorname{argmin}_{\widehat{\mathcal{A}}}(\widehat{E})$, then $u_{s} \in \operatorname{argmin}_{\mathcal{A}}(E)$ for $\mathcal{L}^{1}$-a.e. $s \in(0,1)$ (with $u_{s}$ as in (4.6)).

In particular, if the primal problem $\inf \{E(u): u \in \mathcal{A}\}$ admits a finite number of solutions $\left\{u^{1}, \ldots, u^{k}\right\}$, then

$$
\operatorname{argmin}_{\widehat{\mathcal{A}}}(\widehat{E})=\sum_{i=1}^{k} \theta_{i} \mathbb{1}_{u^{i}}, \quad \theta_{i} \in[0,1],
$$

meaning that $v$ is a piecewise constant function. 
The proof of Theorem 4.1 will be given in Subsection 4.4 after developing the necessary tools in Subsections 4.2 and 4.3 .

4.2. Integral representation of $H$. Let us introduce the one-homogeneous convex integrand $h_{f}$ which will appear in the integral representation of $H$. Such integrand has been already used in several previous works exploiting the classical identification between BV functions and subgraphs of finite perimeter (see for instance [25]). Its definition reads as follows:

Definition 4.2. For $(t, q) \in \mathbb{R} \times \mathbb{R}^{N+1}$, we set:

$$
h_{f}(t, q):= \begin{cases}-q^{t} f\left(t,-q^{x} / q^{t}\right) & \text { if } q^{t}<0 \\ +\infty & \text { if } q^{t}>0 \text { or } q^{t}=0, q^{x} \neq 0 \\ 0 & \text { if }\left(q^{x}, p^{t}\right)=(0,0) .\end{cases}
$$

The above definition will look more natural recalling that it takes its origins in Convex Analysis, as it corresponds precisely to the support function of the epigraph of the Fenchel conjugate $f_{z}^{*}(t, \cdot)$, namely of the set $K(t)$ introduced in (3.15) (see [36, Section 13]). For convenience of the reader, this and the other main properties of $h_{f}$ are stated below.

Lemma 4.3 (properties of $h_{f}$ ). The function $h_{f}$ is lower semicontinuous in $(t, p)$ and convex, positively 1-homogeneous in $p$.

Moreover, $h_{f}(t, \cdot)$ is the support function of the convex set $K(t)$ introduced in (3.15), or equivalently the Fenchel conjugate of the indicatrix function $I_{K(t)}(\cdot)$ (which equals 0 on $K(t)$ and $+\infty$ outside):

$$
h_{f}(t, q)=\sup \{q \cdot \tilde{q}: \tilde{q} \in K(t)\}=I_{K(t)}^{*}(q) .
$$

In particular, the map $t \mapsto K(t)$ defines a lower semicontinuous multifunction (meaning that $\{t \in \mathbb{R}: K(t) \cap A \neq \emptyset\}$ is open for every open subset $A$ of $\left.\mathbb{R}^{N+1}\right)$.

Proof. Since by assumption $f$ is lower semicontinuous in $(t, z)$, it is clear that $h_{f}$ is l.s.c. at any $(t, q)$ with $q^{t}<0$. Let us assume that $q^{t} \geq 0$, and let $\left(t_{n}, q_{n}\right)$ be a sequence converging to $(t, q)$, with $\liminf _{n \rightarrow \infty} h_{f}\left(t_{n}, q_{n}\right)=l \in[0,+\infty)$ (otherwise there is nothing to prove). Then, possibly passing to a subsequence, for every $n$ it holds $q_{n}^{t} \leq 0$, hence $q^{t}=0$. Recalling the growth condition from below satisfied by $f$, we infer that

$$
\left|q_{n}^{t}\right|\left(\alpha\left|\frac{q_{n}^{x}}{q_{n}^{t}}\right|^{p}-r\left(t_{n}\right)\right) \leq l
$$

and therefore also $q^{x}=0$, so that $h_{f}(t, q)=0 \leq l$.

It is immediate from the definition of $h_{f}$ that $h_{f}(t, \cdot)$ is positively 1-homogeneous. The proof of equality (4.16), which in particular implies the convexity of $h_{f}(t, \cdot)$, can be found in [36. Corollary 13.5.1], but for the sake of completeness we sketch it below. By definition, it holds

$$
I_{K(t)}^{*}(p)=\sup \left\{\left(q^{x} \cdot \tilde{q}^{x}+q^{t} \tilde{q}^{t}\right): \tilde{q}^{t} \geq f_{z}^{*}\left(t, \tilde{q}^{x}\right)\right\} .
$$

It is immediately seen the above supremum is 0 in case $q^{t}=\left|q^{x}\right|=0$, and $+\infty$ in case $q^{t}>0$ or $q^{t}=0, q^{x} \neq 0$. In case $p^{t}<0$, it holds

$$
\begin{aligned}
\sup \left\{\left(q^{x} \cdot \tilde{q}^{x}+q^{t} \tilde{q}^{t}\right): \tilde{q}^{t} \geq f_{z}^{*}\left(t, \tilde{q}^{x}\right)\right\} & =\sup \left\{\left(q^{x} \cdot \tilde{q}^{x}+q^{t} f_{z}^{*}\left(t, \tilde{q}^{x}\right)\right): \tilde{q}^{x} \in \mathbb{R}^{N}\right\} \\
& =-q^{t} \sup \left\{\left(-\frac{q^{x}}{q^{t}} \cdot \tilde{q}^{x}-f_{z}^{*}\left(t, \tilde{q}^{x}\right)\right): \tilde{q}^{x} \in \mathbb{R}^{N}\right\} \\
& =-q^{t} f\left(t,-\frac{q^{x}}{q^{t}}\right) .
\end{aligned}
$$


Finally, the lower semicontinuity of the multifunction $t \mapsto K(t)$ follows from [17, Theorem 17].

As a last ingredient, let us recall that one-homogeneous convex integrands such as $h_{f}$ can be integrated in the sense of measures. More precisely, for any bounded vector-valued measure $\lambda \in \mathcal{M}\left(\Omega \times \mathbb{R} ; \mathbb{R}^{N+1}\right)$, the integral of $h_{f}(t, \lambda)$ is meant as

$$
\int_{\Omega \times \mathbb{R}} h_{f}(t, \lambda):=\int_{\Omega \times \mathbb{R}} h_{f}\left(t, \frac{d \lambda}{d|\lambda|}\right) d|\lambda|,
$$

where $|\lambda|$ is the total variation measure of $\lambda$.

Such convex one-homogeneous functional on measures has been studied in [17]. In particular, it can be characterized in terms of the duality $\langle$,$\rangle between \mathcal{M}\left(\Omega \times \mathbb{R} ; \mathbb{R}^{N+1}\right)$ and $\mathcal{C}_{0}\left(\Omega \times \mathbb{R} ; \mathbb{R}^{N+1}\right)$ according to the next lemma:

Lemma 4.4. There holds

$$
\int_{\Omega \times \mathbb{R}} h_{f}(t, \lambda)=\sup \left\{\langle\lambda, \psi\rangle: \psi \in \mathcal{C}_{0}\left(\Omega \times \mathbb{R} ; \mathbb{R}^{N+1}\right), \psi(x, t) \in K(t) \text { on } \Omega \times \mathbb{R}\right\} .
$$

Moreover, the equality above is still true if the supremum at the right hand side is restricted to functions $\psi \in \mathcal{D}\left(\Omega \times \mathbb{R} ; \mathbb{R}^{N+1}\right)$.

Proof. It is easy to check that the supremum at the right hand side of (4.17) is not larger than $\int_{\Omega \times \mathbb{R}} h_{f}(t, \lambda)$. This follows by applying the inequality $h_{f}(t, q) \geq q \cdot \tilde{q}$, holding for every $\tilde{q} \in K(t)$, with $q=\frac{d \lambda}{d|\lambda|}$ and $\tilde{q}=\psi$.

Therefore, the proof of the lemma is concluded if we show that

$$
\int_{\Omega \times \mathbb{R}} h_{f}(t, \lambda)=\sup \{\langle\lambda, \psi\rangle: \psi \in \Sigma\}
$$

with

$$
\Sigma:=\left\{\psi \in \mathcal{D}\left(\Omega \times \mathbb{R} ; \mathbb{R}^{N+1}\right), \psi(x, t) \in K(t) \text { on } \Omega \times \mathbb{R}\right\} .
$$

Clearly, in 4.18) we can replace $\Sigma$ by its closure $\bar{\Sigma}$ (in the uniform norm of $\mathcal{C}_{0}(\Omega \times$ $\left.\mathbb{R} ; \mathbb{R}^{N+1}\right)$ ). Then, according to [17, Theorem 5], in order to prove 44.18) it is enough to establish that

$$
\bar{\Sigma}=\left\{\psi \in \mathcal{C}_{0}\left(\Omega \times \mathbb{R} ; \mathbb{R}^{N+1}\right), \psi(x, t) \in K(t) \text { on } \Omega \times \mathbb{R}\right\}
$$

As $\Sigma$ is $\mathcal{C}^{\infty}$-convex, we may apply [17, Proposition 10], yielding

$$
\bar{\Sigma}=\left\{\psi \in \mathcal{C}_{0}\left(\Omega \times \mathbb{R} ; \mathbb{R}^{N+1}\right), \psi(x, t) \in \Gamma(x, t) \text { on } \Omega \times \mathbb{R}\right\},
$$

with

$$
\Gamma(x, t):=\overline{\{\psi(x, t): \psi \in \Sigma\}} .
$$

Thus we are reduced to prove the equality $K\left(t_{0}\right)=\Gamma\left(x_{0}, t_{0}\right)$ for every $\left(x_{0}, t_{0}\right) \in \Omega \times \mathbb{R}$. Since $K\left(t_{0}\right)$ is closed, it is immediate that $\Gamma\left(x_{0}, t_{0}\right) \subseteq K\left(t_{0}\right)$. Conversely, let $z \in \operatorname{int}\left(K\left(t_{0}\right)\right)$. There exists $\delta>0$ such that, for $\left|t-t_{0}\right|<\delta$, we have $z \in K(t)$ (see [17, Lemma 15]), and consequently the whole interval $[0, z]$ lies in $K(t)$ for $\left|t-t_{0}\right|<\delta$. Then we define $\psi(x, t):=z \alpha(x) \beta_{\delta}(t)$, being $\alpha \in \mathcal{D}(\Omega ;[0,1]), \beta_{\delta} \in \mathcal{D}(\mathbb{R} ;[0,1])$ with $\operatorname{spt}\left(\beta_{\delta}\right) \subset\left[t_{0}-\delta, t_{0}+\delta\right]$, and $\alpha\left(x_{0}\right)=\beta_{0}\left(t_{0}\right)=1$. It is easy to check that the function $\psi$ belongs to $\Sigma$, and hence $z \in \Gamma\left(x_{0}, t_{0}\right)$. Since $\Gamma\left(x_{0}, t_{0}\right)$ is closed, and $K\left(t_{0}\right)$ coincides with the closure of its interior, we have proved that $\Gamma\left(x_{0}, t_{0}\right) \supseteq K\left(t_{0}\right)$. 
We are now ready for the announced integral representation result.

Proposition 4.5 (integral representation of $H)$. For every $v \in B V_{\infty}(\Omega \times \mathbb{R})$, the functional $H$ defined in (4.4) satisfies the equality

$$
H(v)=\int_{\Omega \times \mathbb{R}} h_{f}(t, D v) .
$$

Proof. In view of the definition (4.4) of the functional $H$ and of Lemma 4.4, for every $v \in B V_{\infty}(\Omega \times \mathbb{R})$ there holds

$$
H(v) \geq \sup \left\{\int_{\Omega \times \mathbb{R}} \sigma \cdot D v: \sigma \in \mathcal{K} \cap \mathcal{D}\left(\Omega \times \mathbb{R} ; \mathbb{R}^{N+1}\right)\right\}=\int_{\Omega \times \mathbb{R}} h_{f}(t, D v) .
$$

To obtain also the converse inequality we have to show that, for every $\sigma \in \mathcal{K}$ and every $v \in B V_{\infty}(\Omega \times \mathbb{R})$, there holds $\int_{\Omega \times \mathbb{R}} \sigma \cdot D v \leq \int_{\Omega \times \mathbb{R}} h_{f}(t, D v)$. This is established in the lemma below which completes our proof.

Lemma 4.6 (lower bound for $H$ ). For every $\sigma \in \mathcal{K}$ and every $v \in B V_{\infty}(\Omega \times \mathbb{R})$, there holds

$$
\int_{\Omega \times \mathbb{R}} \sigma \cdot D v \leq \int_{\Omega \times \mathbb{R}} h_{f}(t, D v) .
$$

Proof. The lemma will be obtained by showing separately the following two inequalities:

$$
\begin{gathered}
\int_{\Omega \times(\mathbb{R} \backslash D)} \sigma \cdot D v \leq \int_{\Omega \times(\mathbb{R} \backslash D)} h_{f}(t, D v) \\
\int_{\Omega \times D} \sigma \cdot D v \leq \int_{\Omega \times D} h_{f}(t, D v) .
\end{gathered}
$$

In order to prove (4.19), we need to exploit some facts established in [5, 6] (see also [10]). Recall that, for every $\sigma \in \mathcal{K}$ and $v \in B V_{\infty}(\Omega \times \mathbb{R})$, the measure $\sigma \cdot D v$ defined in 4.2 is absolutely continuous with respect to $|D v|\left(c f\right.$. 4.3.). Moreover, setting $\nu_{v}:=\frac{(\partial D v)}{\partial|D v|}$, the Radon-Nikodym derivative of $\sigma \cdot D v$ with respect to $|D v|$ is given by

$$
\frac{d(\sigma \cdot D v)}{|D v|}=q_{\sigma}\left(x, \nu_{v}\right) \quad|D v| \text {-a.e. in } \Omega \times \mathbb{R},
$$

where $q_{\sigma}: \Omega \times \mathbb{R} \times S^{N} \rightarrow \mathbb{R}$ is the Borel function given by

$$
q_{\sigma}((x, t), \zeta):=\limsup _{\rho \rightarrow 0^{+}} \limsup _{r \rightarrow 0^{+}} \frac{1}{\mathcal{L}^{N+1}\left(C_{r, \rho}((x, t), \zeta)\right)} \int_{C_{r, \rho}((x, t), \zeta)} \sigma(y, s) \cdot \zeta d \mathcal{L}^{N+1},
$$

being

$C_{r, \rho}((x, t), \zeta):=\left\{(y, s) \in \mathbb{R}^{N+1}:|(y-x, s-t) \cdot \zeta| \leq r,|(y-x, s-t)-((y-x, s-t) \cdot \zeta) \zeta| \leq \rho\right\}$.

In view of 4.21, we can rewrite (4.19) as

$$
\int_{\Omega \times(\mathbb{R} \backslash D)} q_{\sigma}\left((x, t), \nu_{v}\right) d|D v| \leq \int_{\Omega \times(\mathbb{R} \backslash D)} h_{f}\left(t, \nu_{v}\right) d|D v| .
$$

We observe that

$$
q_{\sigma}\left(\left(x_{0}, t_{0}\right), \zeta\right) \leq h_{f}^{+}\left(t_{0}, \zeta\right):=\limsup _{t \rightarrow t_{0}} h_{f}(t, \zeta) \quad \forall\left(x_{0}, t_{0}\right) \in \Omega \times \mathbb{R}, \forall \zeta \in S^{N} .
$$

Namely, since $\sigma$ satisfies condition (3.9) (or equivalently (3.13)), by Lemma 4.3 it holds

$$
\sigma(x, t) \cdot \zeta \leq h_{f}(t, \zeta) \quad \text { for } \mathcal{L}^{N+1} \text {-a.e. }(x, t) \in \Omega \times \mathbb{R} .
$$


By taking the mean value over the cylinder $C_{r, \rho}\left(\left(x_{0}, t_{0}\right), \zeta\right)$, and passing to the limsup as $\rho$ and $r$ converge to zero, we obtain

$$
\begin{aligned}
q_{\sigma}\left(\left(x_{0}, t_{0}\right), \zeta\right) & \leq \limsup _{\rho \rightarrow 0^{+}} \limsup _{r \rightarrow 0^{+}} \frac{1}{\mathcal{L}^{N+1}\left(C_{r, \rho}\left(\left(x_{0}, t_{0}\right), \zeta\right)\right)} \int_{C_{r, \rho}\left(\left(x_{0}, t_{0}\right), \zeta\right)} h_{f}(t, \zeta) d \mathcal{L}^{N+1} \\
& \leq h_{f}^{+}\left(t_{0}, \zeta\right) .
\end{aligned}
$$

Now we notice that, thanks to our hypothesis (2.8), we have

$$
h_{f}^{+}(t, \zeta)=h_{f}(t, \zeta) \quad \forall t \in \mathbb{R} \backslash D, \forall \zeta \in S^{N} .
$$

The required inequality (4.22) follows from 4.23) and 4.24).

Let us now prove inequality 4.20). To that aim it is enough to show that $\frac{d\left(D_{x} v\right)}{d|D v|}=0$ $|D v|$-a.e. on $\Omega \times D$, or equivalently that

$$
\nu_{v}=-e_{N+1} \quad|D v| \text {-a.e. on } \Omega \times D .
$$

Indeed in this case, by exploiting condition (3.10) (or equivalently (3.14)), we obtain

$$
\sigma(x, t) \cdot\left(-e_{N+1}\right)=-\sigma^{t}(x, t) \leq f(t, 0)=h_{f}\left(t,-e_{N+1}\right) \forall t \in D \text { and for } \mathcal{L}^{N} \text {-a.e. } x \in \Omega \text {. }
$$

To prove (4.25) we simply observe that

$$
0=\int_{D}\left(\int_{\Omega}\left|D_{x} v(\cdot, t)\right|\right) d t=\int_{\Omega \times D}\left|D_{x} v\right|,
$$

where the first equality follows from the assumption $\mathcal{L}^{1}(D)=0$, and the second one from the slicing formula for $B V$ functions (see [4, Section 3.11]).

Remark 4.7. As one can easily check by inspection of the proof of Lemma 4.6, the inequality (4.38) can be strengthened into

$$
\int_{\omega} f(u, \nabla u) d x=\int_{G_{u} \cap(\omega \times \mathbb{R})} h_{f}\left(t, \nu_{u}\right) d \mathcal{H}^{N} \geq \int_{G_{u} \cap(\omega \times \mathbb{R})} \sigma \cdot \nu_{u} d \mathcal{H}^{N} \quad \forall \omega \text { Borel set } \subset \Omega \text {. }
$$

By the arbitrariness of the Borel set $\omega$ we infer that, for all $u \in \mathcal{A}$ and $\sigma \in \mathcal{B}$, there holds

$$
h_{f}\left(t, \nu_{u}\right) \geq \sigma \cdot \nu_{u} \quad \mathcal{H}^{N} \text {-a.e. on } G_{u} .
$$

Consequently, we see that the validity of inequality (3.10) is extended for free also to values $t \in \mathbb{R} \backslash D$. Indeed, by taking locally constant functions $u$ in $(4.26)$ we obtain that, for all $\sigma \in \mathcal{B}$, there holds $f(t, 0) \geq-\sigma^{t}(x, t)$ for every $t \in \mathbb{R}$ and $\mathcal{L}^{N}$-a.e. $x \in \Omega$.

4.3. Generalized coarea formula. Let $A$ be an open subset of $\mathbb{R}^{d}$. For every function $v \in L_{\text {loc }}^{1}(A)$ and every $s \in \mathbb{R}$, let $\chi_{\{v>s\}}$ denote the characteristic function of the set $\{v>s\}$, i.e.

$$
\chi_{\{v>s\}}(x):= \begin{cases}1 & \text { if } v(x)>s \\ 0 & \text { if } v(x) \leq s .\end{cases}
$$

Following a terminology introduced in [37, we give the following

Definition 4.8. We say that a functional $J: L_{\text {loc }}^{1}(A) \rightarrow[0,+\infty]$ satisfies the generalized coarea formula if for every $u \in L_{\mathrm{loc}}^{1}(A)$ the function $t \mapsto J\left(\chi_{\{v>s\}}\right)$ is Lebesgue-measurable on $\mathbb{R}$ and there holds

$$
J(v)=\int_{-\infty}^{+\infty} J\left(\chi_{\{v>s\}}\right) d s \quad \forall v \in L_{\mathrm{loc}}^{1}(A) .
$$


Remark 4.9. It is readily seen that the following conditions are necessary in order that a functional $J: L_{\text {loc }}^{1}(A) \rightarrow[0,+\infty]$ satisfies the generalized coarea formula:

- $J$ is positively 1-homogeneous (i.e. $J(\lambda v)=\lambda J(v)$ for all $v \in L_{\mathrm{loc}}^{1}(A)$ and $\left.\lambda \geq 0\right)$

$-J\left(\chi_{A}\right)=0$.

Indeed, the 1-homogeneity is immediately obtained via a change of variable in (4.27), whereas the second property follows by applying (4.27) to $v=\chi_{A}$, which gives $J\left(\chi_{A}\right)=$ $\int_{-\infty}^{1} J\left(\chi_{A}\right) d t$

The next result establishes sufficient conditions in order that a functional $J$ satisfies the generalized coarea formula. Its proof is postponed to Section 7 .

Theorem 4.10. (generalized coarea formula) Let $J: L_{\text {loc }}^{1}(A) \rightarrow[0,+\infty]$ be positively 1 -homogeneous and such that $J\left(\chi_{A}\right)=0$. Assume in addition that $J$ is convex, lower semicontinuous, and satisfies the following property: if $\left\{\alpha_{i}\right\}_{1 \leq i \leq k}$ is a family of functions in $\mathcal{C}^{\infty}(\mathbb{R} ;[0,1])$ with $\sum_{i=1}^{k} \alpha_{i} \equiv 1$, setting $\beta_{i}(t):=\int_{0}^{t} \alpha_{i}(s) d s$, it holds

$$
\sum_{i=1}^{k} J\left(\beta_{i} \circ v\right) \leq J(v) \quad \forall v \in L_{\mathrm{loc}}^{1}(A) .
$$

Then $J$ satisfies the generalized coarea formula.

Remark 4.11. It is easy to check that the functional $J: L_{\text {loc }}^{1}(A) \rightarrow[0,+\infty]$ defined by $\int_{A}|D v|$ if $u \in B V(A)$ and $+\infty$ otherwise fulfills all the hypotheses of Theorem 4.10 . Hence $J$ satisfies the generalized coarea formula, which allows to recover the classical coarea formula $\int_{A}|D v|=\int_{-\infty}^{+\infty} \operatorname{Per}(\{v>t\}) d t$ holding for every function $u \in B V(A)$ (see e.g. [4, p.145]).

Theorem 4.10 applies in particular to the functional $H$, as stated below.

Proposition 4.12. (coarea formula for $H$ ) The functional $H$ satisfies the generalized coarea formula.

Proof. Let us check that $H$ satisfies all the assumptions of Theorem 4.10, It is clear from definition (4.4) that $H$ is positively 1-homogeneous, convex, lower semicontinuous, and satisfies $H\left(\chi_{\Omega \times \mathbb{R}}\right)=0$. It remains to check that, if $\left\{\alpha_{i}\right\}_{1 \leq i \leq k}$ is a family of functions in $\mathcal{C}^{\infty}(\mathbb{R} ;[0,1])$ with $\sum_{i=1}^{k} \alpha_{i} \equiv 1$, and $\beta_{i}(t):=\int_{0}^{t} \alpha_{i}(s) d s$, the inequality 4.28 holds. To that aim we may assume without loss of generality that $H(v)<+\infty$, namely that $v \in B V_{\infty}(\Omega \times \mathbb{R})$. We observe that $v \in B V_{\infty}(\Omega \times \mathbb{R})$ implies $\chi_{\{v>s\}} \in B V_{\infty}(\Omega \times \mathbb{R})$ for $\mathcal{L}^{1}$-a.e. $s \in \mathbb{R}$. Then, according to Proposition 4.5 , we have to prove that

$$
\int_{\Omega \times \mathbb{R}} h_{f}(t, D v) \geq \sum_{i=1}^{k} \int_{\Omega \times \mathbb{R}} h_{f}\left(t, D\left(\beta_{i} \circ v\right)\right) .
$$

Denoting by $\widetilde{D} v$ the diffuse part of the measure $D v$ (namely the sum of the absolutely continuous part plus the Cantor part), by the chain rule formula [4, Theorem 3.96], for every $i=1, \ldots, k$, the function $\beta_{i}(v)$ belongs to $B V_{\infty}(\Omega \times \mathbb{R})$ and it holds

$$
D\left(\beta_{i}(v)\right)=\alpha_{i}(v) \widetilde{D} v+\left(\int_{v^{-}(x, t)}^{v^{+}(x, t)} \alpha_{i}(s) d s\right) \nu_{v}(x, t) d \mathcal{H}^{N}\left\llcorner J_{v} .\right.
$$


Then, since $\alpha_{i}$ are nonnegative functions and $h_{f}(t, \cdot)$ is positively 1 -homogeneous, we have $\int_{\Omega \times \mathbb{R}} h_{f}\left(t, D\left(\beta_{i} \circ v\right)\right)=\int_{\Omega \times \mathbb{R}} \alpha_{i}(v) h_{f}(t, \widetilde{D} v)+\int_{J_{v}}\left(\int_{v^{-}(x, t)}^{v^{+}(x, t)} \alpha_{i}(s) d s\right) h_{f}\left(t, \nu_{v}(x, t)\right) d \mathcal{H}^{N}$.

Summing over $i$, and recalling that $\sum_{i=1}^{k} \alpha_{i} \equiv 1$, we deduce that 4.29 is satisfied with equality sign. Thus 4.28 holds, we are in a position to apply Theorem 4.10 , and we obtain that $H$ satisfies the generalized coarea formula.

4.4. Proof of Theorem 4.1. We are going to prove the theorem in two steps. In the first step we prove the equality (4.12), and in the second one we prove the equality (4.13) and the subsequent part of the statement. For the second step we need a slicing formula for the functional $\widehat{E}$ (stated in Proposition 4.13 below), which is obtained thanks to the the coarea formula for $H$ proved in the previous subsection.

Step 1 (Proof of 4.12). Let us show separately the two equalities

$$
\begin{gathered}
\int_{\Omega \times \mathbb{R}} h_{f}\left(t, D \mathbb{1}_{u}\right)= \begin{cases}\int_{\Omega} f(u, \nabla u) d x & \text { if } u \in W^{1, p}(\Omega) \\
+\infty & \text { if } u \in B V(\Omega) \backslash W^{1, p}(\Omega)\end{cases} \\
\int_{\Gamma_{1} \times \mathbb{R}}\left(\mathbb{1}_{u}-v_{0}\right) \gamma^{\prime}(t) d \mathcal{H}^{N-1}(x)=\int_{\Gamma_{1}} \gamma(u) d \mathcal{H}^{N-1}(x) \quad \forall u \in B V(\Omega) .
\end{gathered}
$$

In order to show 4.30 it will be useful to recall few basic facts about subgraphs of $B V$ functions. For any $u \in B V(\Omega)$, the singular set of $\mathbb{1}_{u}$, or equivalently the measure theoretic boundary of the subgraph of $u$, is called the complete graph of $u$, and is denoted by $\Gamma_{u}$. Moreover, we set $\nu_{\Gamma_{u}}$ the inward unit normal to $\Gamma_{u}$. In particular, we have

$$
D \mathbb{1}_{u}=\nu_{\Gamma_{u}} d\left(\mathcal{H}^{N}\left\llcorner\Gamma_{u}\right),\right.
$$

and

$$
\int_{\Omega \times \mathbb{R}} h_{f}\left(t, D \mathbb{1}_{u}\right)=\int h_{f}\left(t, \nu_{\Gamma_{u}}\right) d\left(\mathcal{H}^{N}\left\llcorner\Gamma_{u}\right) .\right.
$$

By writing $D \mathbb{1}_{u}$ as the sum of the two measures

$$
D \mathbb{1}_{u}\left\llcorner( J _ { u } \times \mathbb { R } ) \quad \text { and } \quad D \mathbb { 1 } _ { u } \left\llcorner\left(\left(\Omega \backslash J_{u}\right) \times \mathbb{R}\right),\right.\right.
$$

where $J_{u}$ denotes the jump set of $u$, one obtains a decomposition of $\Gamma_{u}$ into a "vertical part" plus an "approximately continuous part". On the vertical part, $\nu_{\Gamma_{u}}$ is horizontal, and precisely it is given by

$$
\nu_{\Gamma_{u}}(x, t)=\left(\nu_{J_{u}}(x), 0\right) .
$$

On the approximately continuous part, denoting by $u_{+}(x)=\operatorname{aplimsup}_{y \rightarrow x} u(y), \nu_{\Gamma_{u}}$ is given by

$$
\nu_{\Gamma_{u}}\left(x, u_{+}(x)\right)=\frac{(\nabla u(x),-1)}{\sqrt{1+|\nabla u(x)|^{2}}}
$$

if $u$ is approximately differentiable at $x$ (with approximate gradient $\nabla u(x)$ ), and it is horizontal otherwise (namely at points corresponding to the Cantor part of $D u$ ). We refer to [29, Section 4.1.5] for a detailed account of these properties.

In particular, when dealing with functions $u \in W^{1, p}(\Omega)$, the complete graph $\Gamma_{u}$ agrees with the usual graph $G_{u}$, and $\nu_{u}(x, u(x))=\nu_{\Gamma_{u}}\left(x, u_{+}(x)\right)$. 
Then, from the explicit expression 4.15) of $h_{f}$ and the fact that $\nu_{\Gamma_{u}}$ is horizontal except at point $(x, u(x))$ where $u$ is approximately differentiable, we see that $\int_{\Omega \times \mathbb{R}} h_{f}\left(t, D \mathbb{1}_{u}\right)$ is finite only if $u \in W^{1,1}(\Omega)$. In this case, the measure $D \mathbb{1}_{u}$ is given by $\nu_{u} \mathcal{H}^{N}\left\llcorner G_{u}\right.$, and we have

$$
\int_{\Omega \times \mathbb{R}} h_{f}\left(t, D \mathbb{1}_{u}\right)=\int_{\Gamma_{u}} h_{f}\left(t, \nu_{u}\right) d \mathcal{H}^{N} .
$$

Since the Jacobian of the mapping $\Omega \ni x \mapsto(x, u(x)) \in \Gamma_{u}$ is given by $\sqrt{1+|\nabla u|^{2}}$ and since $h_{f}(t, \cdot)$ is positively 1-homogeneous, via change of variable we get

$$
\int_{G_{u}} h_{f}\left(t, \nu_{u}\right) d \mathcal{H}^{N}=\int_{\Omega} h_{f}(u(x),(\nabla u(x),-1)) d x .
$$

Now, by using the definition 4.15 of $h_{f}$, it is immediate to check that the r.h.s. of the above equality agrees with $\int_{\Omega} f(u, \nabla u) d x$, which yields 4.30 .

The identity

$$
\left(\mathbb{1}_{u}-v_{0}\right)= \begin{cases}\mathbb{1}_{[0, u(x)]} & \text { if } u(x)>0 \\ -\mathbb{1}_{[u(x), 0]} & \text { if } u(x)<0 .\end{cases}
$$

together with $\gamma(0)=0$, yields

$$
\int_{\mathbb{R}}\left(\mathbb{1}_{u}-v_{0}\right) \gamma^{\prime}(t) d t=\gamma(u) .
$$

We obtain (4.31) after an integration over $\Gamma_{1}$. The identity (4.12) follows by adding (4.30) and 4.31.

Proposition 4.13. For every $v \in \widehat{\mathcal{A}}$ such that $\widehat{E}(v)<+\infty$, there holds

$$
\widehat{E}(v)=\int_{0}^{1} \widehat{E}\left(\chi_{\{v>s\}}\right) d s=\int_{0}^{1} \widehat{E}\left(\mathbb{1}_{u_{s}}\right) d s=\int_{0}^{1} E\left(u_{s}\right) d s .
$$

Proof. Let $v \in \widehat{\mathcal{A}}$ be such that $\widehat{E}(v)<+\infty$. We claim that it holds

$$
\ell(v)=\int_{0}^{1} \ell\left(\chi_{\{v>s\}}\right) d s=\int_{0}^{1} \ell\left(\mathbb{1}_{u_{s}}\right) d s=\int_{0}^{1} \int_{\Gamma_{1}} \gamma\left(u_{s}\right) d \mathcal{H}^{N-1} d s .
$$

Notice that the second and the third equalities in 4.36 are satisfied in view of 4.7 and (4.12). Thus we have just to prove the first equality, which can be rewritten as

$$
\int_{\Gamma_{1} \times \mathbb{R}} \gamma^{\prime}(t)\left(v-v_{0}\right) d \mathcal{H}^{N-1} d t=\int_{0}^{1}\left\{\int_{\Gamma_{1} \times \mathbb{R}} \gamma^{\prime}(t)\left(\chi_{\{v>s\}}-v_{0}\right) d \mathcal{H}^{N-1} d t\right\} d s .
$$

We write

$$
\begin{aligned}
& \ell(v)=\int_{\Gamma_{1} \times \mathbb{R}_{+}} v(x, t) \gamma^{\prime}(t) d \mathcal{H}^{N-1} d t-\int_{\Gamma_{1} \times \mathbb{R}_{-}}[1-v(x, t)] \gamma^{\prime}(t) d \mathcal{H}^{N-1} d t \\
& \ell\left(\chi_{\{v>s\}}\right)=\int_{\Gamma_{1} \times \mathbb{R}_{+}} \chi_{\{v>s\}}(x, t) \gamma^{\prime}(t) d \mathcal{H}^{N-1} d t-\int_{\Gamma_{1} \times \mathbb{R}_{-}}\left[1-\chi_{\{v>s\}}(x, t)\right] \gamma^{\prime}(t) d \mathcal{H}^{N-1} d t .
\end{aligned}
$$

Now we observe that, since $v-v_{0} \in L^{1}(\Omega \times \mathbb{R})$, and $v(x, \cdot)$ is nonincreasing, $v$ takes values into $[0,1]$. Any function $w$ with values in $[0,1]$ can be written as $w(x)=\int_{0}^{1} \chi_{\{w>s\}} d s$ 
(which is commonly called layer cake representation formula). Then, by applying Fubini Theorem separately to the integrals over $\Gamma_{1} \times \mathbb{R}_{+}$and over $\Gamma_{1} \times \mathbb{R}_{-}$, we have:

$$
\begin{aligned}
\int_{\Gamma_{1} \times \mathbb{R}_{+}} v(x, t) \gamma^{\prime}(t) d \mathcal{H}^{N-1} d t & =\int_{0}^{1} d s \int_{\Gamma_{1} \times \mathbb{R}_{+}} \chi_{\{v>s\}}(x, t) \gamma^{\prime}(t) d \mathcal{H}^{N-1} d t \\
\int_{\Gamma_{1} \times \mathbb{R}_{-}}[1-v(x, t)] \gamma^{\prime}(t) d \mathcal{H}^{N-1} d t & =\int_{0}^{1} d \tau \int_{\Gamma_{1} \times \mathbb{R}_{-}} \chi_{\{1-v>\tau\}}(x, t) \gamma^{\prime}(t) d \mathcal{H}^{N-1} d t \\
& =\int_{0}^{1} d s \int_{\Gamma_{1} \times \mathbb{R}_{-}}\left[1-\chi_{\{v>s\}}(x, t)\right] \gamma^{\prime}(t) d \mathcal{H}^{N-1} d t
\end{aligned}
$$

and we obtain (4.36) by addition.

We are now ready to conclude. By using Proposition 4.12, the equality (4.36), the fact that (as noticed above) $v$ takes values into $[0,1]$, and the equalities $H(0)=H\left(\chi_{\Omega \times \mathbb{R}}\right)=0$, we obtain

$$
\begin{aligned}
\widehat{E}(v)=H(v)+\ell(v) & =\int_{-\infty}^{+\infty} H\left(\chi_{\{v>s\}}\right) d s+\int_{0}^{1} \ell\left(\chi_{\{v>s\}}\right) d s \\
& =\int_{0}^{1} H\left(\chi_{\{v>s\}}\right) d s+\int_{0}^{1} \ell\left(\chi_{\{v>s\}}\right) d s \\
& =\int_{0}^{1} \widehat{E}\left(\chi_{\{v>s\}}\right) d s .
\end{aligned}
$$

Finally, recalling the equalities 4.7) and 4.12), we obtain

$$
\widehat{E}(v)=\int_{0}^{1} \widehat{E}\left(\mathbb{1}_{u_{s}}\right) d s=\int_{0}^{1} E\left(u_{s}\right) d s .
$$

Step 2 (Proof of 4.13) and of last part in Theorem 4.1). Set for brevity

$$
\mathcal{I}=\inf \{E(u): u \in \mathcal{A}\} \quad \text { and } \quad \mathcal{J}:=\inf \{\widehat{E}(v): v \in \widehat{\mathcal{A}}\} .
$$

For every $u \in \mathcal{A}$, the function $v=\mathbb{1}_{u}$ belongs to $\widehat{\mathcal{A}}$. Therefore, in view of the equality (4.12), we immediately see that the inequality $\mathcal{I} \geq \mathcal{J}$ is satisfied.

Conversely, let $v \in \widehat{\mathcal{A}}$ be such that $\widehat{E}(v)<+\infty$. For such a function $v$, the slicing formula (4.35) holds. Such equality implies in particular that, for $\mathcal{L}^{1}$-a.e. $s \in(0,1), u_{s}$ lies in $W^{1, p}(\Omega)$; moreover, since $v=\mathbb{1}_{u_{0}}$ on $\Gamma_{0} \times \mathbb{R}$, it holds $u_{s}=u_{0}$ on $\Gamma_{0}$. Therefore, for $\mathcal{L}^{1}$-a.e. $s \in(0,1)$, we have $u_{s} \in \mathcal{A}$, which implies $E\left(u_{s}\right) \geq \mathcal{I}$. After an integration over $(0,1)$, by (4.35), we obtain $\widehat{E}(v) \geq \mathcal{I}$. By the arbitrariness of $v \in \widehat{\mathcal{A}}$, we conclude that $\mathcal{J} \geq \mathcal{I}$. The equalities (4.12) and (4.13) imply immediately that, if $u \in \operatorname{argmin}_{\mathcal{A}}(E)$, then $\mathbb{1}_{u} \in$ $\operatorname{argmin}_{\widehat{\mathcal{A}}}(\widehat{E})$. Since we know from Proposition 2.2 that the infimum $\mathcal{I}$ is finite and attained, we deduce that the same holds true for the infimum $\mathcal{J}$.

Finally, if $v \in \operatorname{argmin}_{\widehat{\mathcal{A}}}(\widehat{E}), 4$ (4.35) and 4.13 imply that $u_{s} \in \operatorname{argmin}_{\mathcal{A}}(E)$ for $\mathcal{L}^{1}$-a.e. $s \in(0,1)$. In particular this assertion implies that, in case the primal problem has a finite number of solutions, $v$ must be a convex combination of them as stated in 4.14. 
4.5. Proof of the inequality $\mathcal{I} \geq \mathcal{I}^{*}$ in Theorem 3.4. We are going to prove that, for every $u \in \mathcal{A}$ and $\sigma \in \mathcal{B}$, there holds

$$
\begin{aligned}
\int_{\Omega} f(u, \nabla u) d x+\int_{\Gamma_{1}} \gamma(u) d \mathcal{H}^{N-1} & \geq \int_{G_{u}} \sigma \cdot \nu_{u} d \mathcal{H}^{N}+\int_{\Gamma_{1}} \gamma(u) d \mathcal{H}^{N-1} \\
& =\int_{G_{u_{0}}} \sigma \cdot \nu_{u_{0}} d \mathcal{H}^{N}+\int_{\Gamma_{1}} \gamma\left(u_{0}\right) d \mathcal{H}^{N-1} .
\end{aligned}
$$

Once proved (4.37), by passing to the infimum over $u \in \mathcal{A}$ and to the supremum over $\sigma \in \mathcal{B}$ respectively at the left hand side and at the right hand side, we obtain the inequality $\mathcal{I} \geq \mathcal{I}^{*}$.

Let us prove separately the inequality in the first line of (4.37), and the equality in the second line.

The inequality in the first line of (4.37) follows simply by recalling (4.30) and applying Lemma 4.6 with $v=\mathbb{1}_{u}$ :

$$
\int_{\Omega} f(u, \nabla u) d x=\int_{G_{u}} h_{f}\left(t, \nu_{u}\right) d \mathcal{H}^{N} \geq \int_{G_{u}} \sigma \cdot \nu_{u} d \mathcal{H}^{N} .
$$

The equality in the second line of 4.37 follows via an integration by parts formula that we state separately in the next lemma, since it will be useful again in the sequel. It is obtained as an application of the following generalized divergence theorem, that we recall from [6] (see also [10]): for every $\sigma \in X_{1}(\Omega \times \mathbb{R})$ and every $v \in B V_{\infty}(\Omega \times \mathbb{R}) \cap L^{1}(\Omega \times \mathbb{R})$, there holds

$$
\int_{\Omega \times \mathbb{R}} \sigma \cdot D v+\int_{\Omega \times \mathbb{R}} v \operatorname{div} \sigma d x=\int_{\partial \Omega \times \mathbb{R}}\left(\sigma \cdot \nu_{\Omega}\right) v d \mathcal{H}^{N} .
$$

Notice that the boundary integral at the r.h.s. is well-defined since the normal trace $\sigma \cdot \nu_{\Omega}$ is in $L^{\infty}(\partial \Omega \times \mathbb{R})$, and the function $v$ is in $L^{1}(\partial \Omega \times \mathbb{R})$ because $v \in B V(\Omega \times \mathbb{R})$.

Lemma 4.14. (an integration by parts formula) For every $\sigma$ in $X_{1}(\Omega \times \mathbb{R})$ satisfying (3.12) and (3.11), and every $v$ in the class $\widehat{\mathcal{A}}$ defined in (4.9), there holds

$$
\int_{\Omega \times \mathbb{R}} \sigma \cdot D v+\int_{\Gamma_{1} \times \mathbb{R}} \gamma^{\prime}(t)\left(v-v_{0}\right) d \mathcal{H}^{N-1} d t=\int_{G_{u_{0}}} \sigma \cdot \nu_{u_{0}} d \mathcal{H}^{N}+\int_{\Gamma_{1}} \gamma\left(u_{0}\right) d \mathcal{H}^{N-1}
$$

In particular, if $v$ is of the form $v=\mathbb{1}_{u}$ for some $u \in \mathcal{A}$, we obtain

$$
\int_{G_{u}} \sigma \cdot \nu_{u} d \mathcal{H}^{N}+\int_{\Gamma_{1}} \gamma(u) d \mathcal{H}^{N-1}=\int_{G_{u_{0}}} \sigma \cdot \nu_{u_{0}} d \mathcal{H}^{N}+\int_{\Gamma_{1}} \gamma\left(u_{0}\right) d \mathcal{H}^{N-1} .
$$

Proof. For every $\sigma$ and $v$ as in the assumptions, we have that the function $v-\mathbb{1}_{u_{0}}=$ $\left(v-v_{0}\right)+\left(v_{0}-\mathbb{1}_{u_{0}}\right)$ is in $B V_{\infty}(\Omega \times \mathbb{R}) \cap L^{1}(\Omega \times \mathbb{R})$, and $\sigma$ is in $X_{1}(\Omega \times \mathbb{R})$. Therefore, we are in a position to apply the generalized Gauss-Green formula 4.39 ). Exploiting also the condition $\operatorname{div} \sigma=0$ in $\Omega \times \mathbb{R}$, we obtain

$$
\begin{aligned}
\int_{\Omega \times \mathbb{R}} \sigma \cdot\left(D v-D \mathbb{1}_{u_{0}}\right) & =\int_{\partial \Omega \times \mathbb{R}}\left(\sigma^{x} \cdot \nu_{\Omega}\right)\left(v-\mathbb{1}_{u_{0}}\right)=-\int_{\Gamma_{1} \times \mathbb{R}} \gamma^{\prime}(t)\left(v-\mathbb{1}_{u_{0}}\right) d \mathcal{H}^{N-1} d t \\
& =-\int_{\Gamma_{1} \times \mathbb{R}} \gamma^{\prime}(t)\left[\left(v-v_{0}\right)-\left(\mathbb{1}_{u_{0}}-v_{0}\right)\right] d \mathcal{H}^{N-1} d t .
\end{aligned}
$$


Hence,

$$
\begin{aligned}
& \int_{\Omega \times \mathbb{R}} \sigma \cdot D v+\int_{\Gamma_{1} \times \mathbb{R}} \gamma^{\prime}(t)\left(v-v_{0}\right) d \mathcal{H}^{N-1} d t \\
= & \int_{\Omega \times \mathbb{R}} \sigma \cdot D \mathbb{1}_{u_{0}}+\int_{\Gamma_{1} \times \mathbb{R}} \gamma^{\prime}(t)\left(\mathbb{1}_{u_{0}}-v_{0}\right) d \mathcal{H}^{N-1} d t \\
= & \int_{G_{u_{0}}} \sigma \cdot \nu_{u_{0}} d \mathcal{H}^{n-1}+\int_{\Gamma_{1}} \gamma\left(u_{0}\right) d \mathcal{H}^{N-1} .
\end{aligned}
$$

Notice that in the last equality we have used the identity $\int_{\mathbb{R}} \gamma^{\prime}(t)\left(\mathbb{1}_{u_{0}}-v_{0}\right) d t=\gamma\left(u_{0}\right)$, already shown in the proof of Theorem 4.1 ( $c f$. equation 4.34$)$ ).

We have thus completed the proof of (4.37) and hence of the inequality $\mathcal{I} \geq \mathcal{I}^{*}$ in Theorem 3.4 .

\section{Optimality CONDITIONS AND Min-MAX FORMUlATiON}

Out next goal is to provide necessary and sufficient conditions for optimality:

Theorem 5.1. (geometric optimality condition) Let $u \in \mathcal{A}$ and $\sigma \in \mathcal{B}$. Then $u$ is a solution to the primal problem problem $(\mathcal{P})$ in $(2.1)$ and $\sigma$ is a solution to the dual problem $\left(\mathcal{P}^{*}\right)$ in $(3.16)$ if and only if

$$
h_{f}\left(t, \nu_{u}\right)=\sigma \cdot \nu_{u} \quad \mathcal{H}^{N} \text {-a.e. on } G_{u} .
$$

In this case, we say that $\sigma$ is a calibration for $u$.

Proof. Assume that $u \in \mathcal{A}$ and $\sigma \in \mathcal{B}$ satisfy (5.1). By using in the order the definition of $\mathcal{I}^{*}$, Lemma 4.14, condition (5.1), the equality (4.30), and the definition of $\mathcal{I}$, we obtain

$$
\begin{aligned}
\mathcal{I}^{*} & \geq \int_{G_{u_{0}}} \sigma \cdot \nu_{u_{0}} d \mathcal{H}^{N}+\int_{\Gamma_{1}} \gamma\left(u_{0}\right) d \mathcal{H}^{N-1}=\int_{G_{u}} \sigma \cdot \nu_{u} d \mathcal{H}^{N}+\int_{\Gamma_{1}} \gamma(u) d \mathcal{H}^{N-1} \\
& =\int_{G_{u}} h_{f}\left(t, \nu_{u}\right) d \mathcal{H}^{N}+\int_{\Gamma_{1}} \gamma(u) d \mathcal{H}^{N-1}=\int_{\Omega} f(u, \nabla u) d x+\int_{\Gamma_{1}} \gamma(u) d \mathcal{H}^{N-1} \geq \mathcal{I} .
\end{aligned}
$$

Since we know from Theorem 3.4 that $\mathcal{I}=\mathcal{I}^{*}$, we infer that all the inequalities above hold as equalities, which means in particular that $u$ and $\sigma$ are optimal respectively for the primal and the dual problem.

Assume that $u \in \mathcal{A}$ and $\sigma \in \mathcal{B}$ are optimal respectively for the primal and the dual problem. By using in the order Lemma 4.14, the optimality of $\sigma$, Theorem 3.4, the optimality of $u$, and the equality 4.30 , we obtain

$$
\begin{aligned}
\int_{G_{u}} \sigma \cdot \nu_{u} d \mathcal{H}^{N}+\int_{\Gamma_{1}} \gamma(u) d \mathcal{H}^{N-1} & =\int_{G_{u_{0}}} \sigma \cdot \nu_{u_{0}} d \mathcal{H}^{N}+\int_{\Gamma_{1}} \gamma\left(u_{0}\right) d \mathcal{H}^{N-1}=\mathcal{I}^{*} \\
& =\mathcal{I}=\int_{\Omega} f(u, \nabla u) d x+\int_{\Gamma_{1}} \gamma(u) d \mathcal{H}^{N-1} \\
& =\int_{G_{u}} h_{f}\left(t, \nu_{u}\right) d \mathcal{H}^{N}+\int_{\Gamma_{1}} \gamma(u) d \mathcal{H}^{N-1} .
\end{aligned}
$$

We infer that $\int_{G_{u}} \sigma \cdot \nu_{u} d \mathcal{H}^{N}=\int_{G_{u}} h_{f}\left(t, \nu_{u}\right) d \mathcal{H}^{N}$. In turn, recalling the inequality 4.26 in Remark 4.7, this implies (5.1).

From a practical point of view, in order to construct a calibration, it is useful to rephrase condition (5.1) more explicitly as done in the next result. 
Corollary 5.2 (user's form of optimality conditions). Let $u \in \mathcal{A}$ and $\sigma \in \mathcal{B}$, with $\sigma$ continuous on $\Omega \times(\mathbb{R} \backslash D)$. Then condition (5.1) is satisfied if and only if there holds

$$
\begin{gathered}
\sigma^{x}(x, u(x)) \in \partial_{z} f(u(x), \nabla u(x)) \text { for } \mathcal{L}^{N} \text {-a.e. } x \in u^{-1}(\mathbb{R} \backslash D) ; \\
\sigma^{t}(x, u(x))=f_{z}^{*}\left(u(x), \sigma^{x}(x, u(x))\right) \quad \text { for } \mathcal{L}^{N} \text {-a.e. } x \in u^{-1}(\mathbb{R} \backslash D) ; \\
\sigma^{t}(x, t)=-f(t, 0) \quad \forall t \in \mathbb{R} \text { and for } \mathcal{L}^{N} \text {-a.e. } x \in\{u=t\} .
\end{gathered}
$$

(Note that the set of values $t \in \mathbb{R}$ such that $\mathcal{L}^{N}(\{u=t\})>0$ is at most countable.)

Proof. By (5.2)-(5.3), we infer that the following equality is satisfied $\mathcal{H}^{N}$-a.e. on $G_{u} \cap[\Omega \times$ $(\mathbb{R} \backslash D)]:$

$$
\begin{aligned}
\sigma \cdot \nu_{u} & =\nu_{u}^{x} \cdot \sigma^{x}+\nu_{u}^{t} f_{z}^{*}\left(u, \sigma^{x}\right) \\
& =-\nu_{u}^{t}\left[-\frac{\nu_{u}^{x}}{\nu_{u}^{t}} \cdot \sigma^{x}-f_{z}^{*}\left(u, \sigma^{x}\right)\right] \\
& =-\nu_{u}^{t}\left[\nabla u \cdot \sigma^{x}-f_{z}^{*}\left(u, \sigma^{x}\right)\right] \\
& =-\nu_{u}^{t} f(u, \nabla u) \\
& =-\nu_{u}^{t} f\left(u,-\frac{\nu_{u}^{x}}{\nu_{u}^{t}}\right)=h_{f}\left(t, \nu_{u}\right) .
\end{aligned}
$$

On the other hand, by $(5.4), \mathcal{H}^{N}$-a.e. on $G_{u} \cap[\Omega \times D]$ we have

$$
\sigma \cdot\left(-e_{N+1}\right)=-\sigma^{t}(x, t)=f(t, 0)=h_{f}\left(t,-e_{N+1}\right) .
$$

Recalling (4.25) with $v=\mathbb{1}_{u}$, we conclude that (5.1) is fulfilled.

Conversely, assume that (5.1) holds true.

Since $\sigma$ satisfies (3.9) and is assumed to be continuous on $\Omega \times(\mathbb{R} \backslash D)$, the following chain of inequalities is satisfied $\mathcal{H}^{N}$-a.e. on $G_{u} \cap[\Omega \times(\mathbb{R} \backslash D)]$ :

$$
\begin{aligned}
h_{f}\left(t, \nu_{u}\right) & =\sigma \cdot \nu_{u} \leq \nu_{u}^{x} \cdot \sigma^{x}+\nu_{u}^{t} f_{z}^{*}\left(u, \sigma^{x}\right) \\
& =-\nu_{u}^{t}\left[-\frac{\nu_{u}^{x}}{\nu_{u}^{t}} \cdot \sigma^{x}-f_{z}^{*}\left(u, \sigma^{x}\right)\right] \\
& =-\nu_{u}^{t}\left[\nabla u \cdot \sigma^{x}-f_{z}^{*}\left(u, \sigma^{x}\right)\right] \\
& \leq-\nu_{u}^{t} f(u, \nabla u) \\
& =-\nu_{u}^{t} f\left(u,-\frac{\nu_{u}^{x}}{\nu_{u}^{t}}\right)=h_{f}\left(t, \nu_{u}\right) .
\end{aligned}
$$

We deduce that the two inequalities appearing in the chain are actually equalities, which yields $(5.2)-(5.3)$.

On the other hand, since $\sigma$ satisfies 3.10 on $\Omega \times \mathbb{R}$ (cf. Remark 3.2 , , $\mathcal{H}^{N}$-a.e. on $G_{u} \cap[\Omega \times \mathbb{R}]$ we have

$$
h_{f}\left(t,-e_{N+1}\right)=\sigma \cdot\left(-e_{N+1}\right)=-\sigma^{t}(x, t) \leq f(t, 0)=h_{f}\left(t,-e_{N+1}\right) .
$$

We conclude that the inequality appearing in the line above holds with equality sign, which yields (5.4).

Remark 5.3. In the case when $f$ is differentiable and convex in $(t, z)$ and $\gamma^{\prime} \equiv c$, it is easy to construct an explicit calibration for a given solution $\bar{u}$ to problem $(\mathcal{P})$. Indeed, denoting 
by $\bar{\sigma}$ a solution to the classical dual problem ( $c f$. Remark 3.8), we claim that the field $\sigma$ defined on $\Omega \times \mathbb{R}$ by

$$
\left\{\begin{array}{l}
\sigma^{x}(x, t)=\bar{\sigma}(x) \\
\sigma^{t}(x, t)=f_{z}^{*}(\bar{u}, \bar{\sigma})-(\operatorname{div} \bar{\sigma})(t-\bar{u}(x)),
\end{array}\right.
$$

is a calibration for $\bar{u}$, provided it is continuous on $\Omega \times \mathbb{R}$.

Namely, by classical duality, $\bar{u}$ and $\bar{\sigma}$ satisfy the optimality conditions

$$
\begin{gathered}
\bar{\sigma}=\partial_{z} f(\bar{u}, \nabla \bar{u}), \quad \operatorname{div} \bar{\sigma}=\partial_{t} f(\bar{u}, \nabla \bar{u}) \quad \mathcal{L}^{N} \text {-a.e. in } \Omega \\
\bar{\sigma} \cdot \nu_{\Omega}=-\gamma^{\prime}(\bar{u}) \quad \mathcal{H}^{N-1} \text {-a.e. on } \Gamma_{1} .
\end{gathered}
$$

In view of (5.6) and of the continuity assumption made on $\sigma$, Corollary 5.2 (applied with $D=\emptyset$ ) ensures that $\sigma$ is a calibration for $\bar{u}$, provided we show that $\sigma \in \mathcal{B}$.

It is immediate to verify that $\sigma$ satisfies (3.11). By (5.7) and the assumption $\gamma^{\prime} \equiv c$, it satisfies also (3.12). It only remains to check (3.9), namely

$$
f_{z}^{*}(\bar{u}, \bar{\sigma})-(\operatorname{div} \bar{\sigma})(t-\bar{u}(x)) \geq f_{z}^{*}(t, \bar{\sigma}) \quad \mathcal{L}^{N+1} \text {-a.e. on } \Omega \times \mathbb{R},
$$

or equivalently

$$
f_{z}^{*}(\bar{u}, \bar{\sigma}) \geq \sup _{z \in \mathbb{R}^{N}}[\bar{\sigma} \cdot z-f(t, z)]+(\operatorname{div} \bar{\sigma})(t-\bar{u}(x)) \quad \mathcal{L}^{N+1} \text {-a.e. on } \Omega \times \mathbb{R} .
$$

In turn the latter inequality is satisfied provided

$$
\begin{aligned}
f_{z}^{*}(\bar{u}, \bar{\sigma}) & \geq \sup _{(t, z) \in \mathbb{R}^{N+1}}[(\operatorname{div} \bar{\sigma}, \bar{\sigma}) \cdot(t, z)-f(t, z)]-\bar{u}(x) \operatorname{div} \bar{\sigma} \\
& =f^{*}(\operatorname{div} \bar{\sigma}, \bar{\sigma})-\bar{u}(x) \operatorname{div} \bar{\sigma} \quad \mathcal{L}^{N} \text {-a.e. on } \Omega
\end{aligned}
$$

where $f^{*}$ denotes the global Fenchel conjugate of $f$ with respect to the pair $(t, z)$.

Now, by the two equations in (5.6), we have that $(\operatorname{div} \bar{\sigma}, \bar{\sigma})$ satisfy the Fenchel equality

$$
f^{*}(\operatorname{div} \bar{\sigma}, \bar{\sigma})+f(\bar{u}, \nabla \bar{u})=\bar{u}(x) \operatorname{div} \bar{\sigma}+\nabla \bar{u}(x) \cdot \bar{\sigma} \quad \mathcal{L}^{N} \text {-a.e. on } \Omega .
$$

Inserting this identity into (5.8), we are reduced to

$$
f_{z}^{*}(\bar{u}, \bar{\sigma}) \geq \nabla \bar{u}(x) \cdot \bar{\sigma}-f(\bar{u}, \nabla \bar{u}) \quad \mathcal{L}^{N} \text {-a.e. on } \Omega,
$$

which is satisfied by definition of $f_{z}^{*}$ (and actually holds as an equality since $\bar{\sigma}=\partial_{z} f(\bar{u}, \nabla \bar{u})$ ).

Hereafter we give a min-max formulation of our duality result. For every pair $(v, \sigma)$, with $v \in B V_{\infty}(\Omega \times \mathbb{R})$ and $\sigma \in X_{1}(\Omega \times \mathbb{R})$, we introduce the Lagrangian

$$
L(v, \sigma):=\int_{\Omega \times \mathbb{R}} \sigma \cdot D v+\int_{\Gamma_{1} \times \mathbb{R}} \gamma^{\prime}(t)\left(v-v_{0}\right) d \mathcal{H}^{N-1} .
$$

Theorem 5.4 (saddle point). There holds

$$
\mathcal{I}=\inf _{v \in \widehat{\mathcal{A}}} \sup _{\sigma \in \mathcal{K}} L(v, \sigma)=\sup _{\sigma \in \mathcal{K}} \inf _{v \in \widehat{\mathcal{A}}} L(v, \sigma)=\mathcal{I}^{*} .
$$

Moreover, a pair $(\bar{v}, \bar{\sigma})$ is optimal for the convexified infimum problem $\inf \{\widehat{E}(v): v \in \widehat{\mathcal{A}}\}$ and for the dual problem $\left(\mathcal{P}^{*}\right)$ in (3.16) if and only if it is a saddle point for $L$, namely

$$
L(\bar{v}, \sigma) \leq L(\bar{v}, \bar{\sigma}) \leq L(v, \bar{\sigma}) \quad \forall(v, \sigma) \in \widehat{\mathcal{A}} \times \mathcal{K} .
$$


Remark 5.5. (i) Notice that, since the class $\mathcal{A}$ is not weakly compact, the equality $\mathcal{I}=\mathcal{I}^{*}$ already established in Theorem 3.4 cannot be deduced by applying an inf-sup commutation argument to the bivariate Lagrangian $L$ over the product space $\mathcal{A} \times \mathcal{K}$.

(ii) We emphasize that the class $\mathcal{K}$ appearing in the saddle point problem does not include the divergence free condition. In fact, such condition is handled by duality, through the use of the variable $v$ seen as a Lagrange multiplier. (In analogy with fluid dynamic, one may think of $\sigma$ as the speed of an incompressible fluid, and of $v$ as its pressure).

Proof of Theorem 5.4. Thanks to the equality 4.13 in Theorem 4.1, recalling the definitions 4.11), 4.4 , 4.10), and 5.11) of $\widehat{E}, H, \ell$, and $L$, we obtain

$$
\begin{aligned}
\mathcal{I} & =\inf \{\widehat{E}(v): v \in \widehat{\mathcal{A}}\}=\inf \{H(v)+\ell(v): v \in \widehat{\mathcal{A}}\} \\
& =\inf _{v \in \widehat{\mathcal{A}}} \sup _{\sigma \in \mathcal{K}}\left\{\int_{\Omega \times \mathbb{R}} \sigma \cdot D v+\int_{\Gamma_{1} \times \mathbb{R}} \gamma^{\prime}(t)\left(v-v_{0}\right) d \mathcal{H}^{N-1} d t\right\}=\inf _{v \in \widehat{\mathcal{A}}} \sup _{\sigma \in \mathcal{K}} L(v, \sigma) .
\end{aligned}
$$

Since we know from Theorem 3.4 that $\mathcal{I}=\mathcal{I}^{*}$, in order to complete the proof it remains to show that $\mathcal{I}^{*}=\sup _{\sigma \in \mathcal{K}} \inf _{v \in \widehat{\mathcal{A}}} L(v, \sigma)$. To that aim let us show that, for every $\sigma \in \mathcal{K}$, it holds

$$
\inf _{v \in \widehat{\mathcal{A}}} L(v, \sigma)= \begin{cases}-\infty & \text { if } \sigma \notin \mathcal{B} \\ \int_{G_{u_{0}}} \sigma \cdot \nu_{u_{0}} d \mathcal{H}^{N}+\int_{\Gamma_{1}} \gamma\left(u_{0}\right) d \mathcal{H}^{N-1} & \text { if } \sigma \in \mathcal{B} .\end{cases}
$$

Indeed, the Lagrangian $L(v, \sigma)$ can be rewritten as

$$
L(v, \sigma)=\int_{\Omega \times \mathbb{R}} \sigma \cdot\left(D v-D \mathbb{1}_{u_{0}}\right)+\int_{G_{u_{0}}} \sigma \cdot \nu_{u_{0}} d \mathcal{H}^{N}+\int_{\Gamma_{1} \times \mathbb{R}} \gamma^{\prime}(t)\left(v-v_{0}\right) d \mathcal{H}^{N-1} .
$$

Then, by exploiting the generalized Gauss-Green formula 4.39 , we get

$$
\begin{aligned}
L(v, \sigma) & =-\int_{\Omega \times \mathbb{R}} \operatorname{div} \sigma \cdot\left(v-\mathbb{1}_{u_{0}}\right)+\int_{\Gamma_{0} \times \mathbb{R}}\left(v-\mathbb{1}_{u_{0}}\right)\left(\sigma^{x} \cdot \nu_{\Omega}\right) d \mathcal{H}^{N-1} d t \\
& +\int_{\Gamma_{1} \times \mathbb{R}}\left(v-v_{0}\right)\left(\gamma^{\prime}(t)+\sigma^{x} \cdot \nu_{\Omega}\right) d \mathcal{H}^{N-1} d t \\
& +\int_{\Gamma_{1} \times \mathbb{R}}\left(v_{0}-\mathbb{1}_{u_{0}}\right)\left(\sigma^{x} \cdot \nu_{\Omega}\right) d \mathcal{H}^{N-1} d t+\int_{G_{u_{0}}} \sigma \cdot \nu_{u_{0}} d \mathcal{H}^{N}
\end{aligned}
$$

Now, by taking $v \in \widehat{\mathcal{A}}$ of the form $v=\mathbb{1}_{u_{0}}+\varphi$, with $\varphi \in \mathcal{D}(\Omega \times \mathbb{R})$, we obtain that $\inf _{v \in \widehat{\mathcal{A}}} L(v, \sigma)$ cannot be finite unless $\operatorname{div} \sigma=0$ in $\Omega \times \mathbb{R}$. Next, by taking $v=v_{0}+\varphi$, with $\varphi \in \mathcal{D}(\bar{\Omega} \times \mathbb{R})$ such that $\varphi=0$ on $\Gamma_{0} \times \mathbb{R}$, we see that the normal trace of $\sigma$ must agree with $-\gamma^{\prime}(t)$ on $\Gamma_{1} \times \mathbb{R}$. We conclude that (5.10) is true by recalling (4.34).

The last part of the statement is a standard equivalence in min-max theory (see for instance [26]).

As mentioned in Remark 3.6, whenever the solutions to the primal problem are bounded, we can settle our duality theory on a bounded set of the form $\Omega \times[m, M]$.

For a given $u_{0} \in W^{1, p}\left(\Omega ;[m, M]\right.$, we denote by $\mathcal{I}(m, M)$ and $\mathcal{I}^{*}(m, M)$ respectively the infimum of the primal problem $(\mathcal{P})$ and the supremum of the dual problem $\left(\mathcal{P}^{*}\right)$ over the 
classes $\mathcal{A}(m, M)$ and $\mathcal{B}(m, M)$ introduced in Remark 3.6. Then we set

$$
\begin{aligned}
& \widehat{\mathcal{A}}(m, M):=\{v \in \mathcal{A}: v(x, t)=1 \text { for } t<m, v(x, t)=0 \text { for } t>M\} \\
& \left.\left.\mathcal{K}(m, M):=\left\{\sigma \in X_{1}(\Omega \times(m, M)) \text { satisfying } 3.19\right)-3.20\right)\right\},
\end{aligned}
$$

Accordingly, the Lagrangian $L$ must be now intended as

$$
L(v, \sigma):=\int_{\Omega \times[m, M]} \sigma \cdot D v+\int_{\Gamma_{1} \times[m, M]} \gamma^{\prime}(t)\left(v-v_{0}\right) d \mathcal{H}^{N-1} .
$$

Remark 5.6. Note that in (5.11) the first integral may have a non vanishing contribution on the horizontal part of the boundary (namely the set $\Omega \times\{m, M\}$ ), in case the function $v$ has a jump on such interfaces. More precisely, we have:

$\int_{\Omega \times[m, M]} \sigma \cdot D v=\int_{\Omega \times(m, M)} \sigma \cdot D v+\int_{\Omega}\left[\sigma^{t}(x, M)\left(0-v\left(x, M^{-}\right)\right)+\sigma^{t}(x, m)\left(v\left(x, m^{+}\right)-1\right)\right]$, being $v\left(x, m^{+}\right)$and $v\left(x, M^{-}\right)$respectively the traces of $v$ on $\Omega \times\{m\}$ and $\Omega \times\{M\}$.

We can now reformulate the following variant of Theorems 3.4 and 5.4 .

Proposition 5.7. With the above notation, there holds:

$$
\mathcal{I}(m, M)=\inf _{v \in \widehat{\mathcal{A}}(m, M)} \sup _{\sigma \in \mathcal{K}(m, M)} L(v, \sigma)=\sup _{\sigma \in \mathcal{K}(m, M)} \inf _{v \in \widehat{\mathcal{A}}(m, M)} L(v, \sigma)=\mathcal{I}^{*}(m, M) .
$$

Moreover, a pair $(\bar{v}, \bar{\sigma})$ is optimal for the infimum problem $\inf \{\widehat{E}(v): v \in \widehat{\mathcal{A}}(m, M)\}$ and for the dual problem $\left(\mathcal{P}^{*}\right)$ settled over $\mathcal{B}(m, M)$ if and only if

$$
L(\bar{v}, \sigma) \leq L(\bar{v}, \bar{\sigma}) \leq L(v, \bar{\sigma}) \quad \forall(v, \sigma) \in \widehat{\mathcal{A}}(m, M) \times \mathcal{K}(m, M) .
$$

Proof. The statement can be proved in the analogous way as done for Theorem 5.4, taking into account that, by following the same proof as in Theorem 3.4. one can check that $\mathcal{I}(m, M)=\mathcal{I}^{*}(m, M)$.

\section{Application to a free boundary problem}

6.1. Description of the problem. In this section we illustrate the application of our method to the free boundary problem

$$
\mathcal{I}(\Omega, \lambda):=\inf \left\{\int_{\Omega} \frac{1}{2}|\nabla u|^{2}+\lambda|\{u>0\}|: u \in W^{1,2}(\Omega), u=1 \text { on } \partial \Omega\right\},
$$

which has been firstly considered in the pioneering paper [2].

The free boundary in the minimization problem $\sqrt{6.1)}$ is the frontier of the zero level set $E:=\{u=0\}$. Actually, the infimum $\mathcal{I}(\Omega, \lambda)$ can be recast by solving the shape optimization problem

being $u_{E}$ the solution to

$$
\inf _{E}\left\{\int_{\Omega} \frac{1}{2}\left|\nabla u_{E}\right|^{2}+\lambda|\Omega \backslash E|\right\},
$$

$$
\begin{cases}\Delta u=0 & \text { in } \Omega \backslash E \\ u=0 & \text { in } E \\ u=1 & \text { on } \partial \Omega .\end{cases}
$$


Such problem falls in our setting by choosing

$$
f(t, z)=\frac{1}{2}|z|^{2}+\lambda \chi_{(0,+\infty)}(t), \quad\left(\Gamma_{0}, \Gamma_{1}\right)=(\partial \Omega, \emptyset), \quad u_{0} \equiv 1 .
$$

Notice that the function $f$ satisfies the standing assumptions, and in particular the discontinuity set $D$ appearing in $(2.8)$ is given by $\{t=0\}$.

Then, according to Theorem 3.4, we have $\mathcal{I}(\Omega, \lambda)=\mathcal{I}^{*}(\Omega, \lambda)$. As disclosed in the Introduction, the dual problem reads:

$$
\mathcal{I}^{*}(\Omega, \lambda):=\sup \left\{-\int_{\Omega} \sigma^{t}(x, 1) d x: \sigma \in \mathcal{B}\right\},
$$

where $\mathcal{B}$ is the class of bounded divergence free vector field on $\Omega \times \mathbb{R}$ satisfying the constraints

$$
\sigma^{t}(x, t)+\lambda \geq \frac{1}{2}\left|\sigma^{x}(x, t)\right|^{2} \quad \text { a.e. on } \Omega \times \mathbb{R}, \quad \sigma^{t}(x, 0) \geq 0 \text { a.e. on } \Omega .
$$

It is easy to check that any solution $u \in W^{1,2}(\Omega)$ to problem (6.1) takes values in $[0,1]$. Therefore, according Remark 3.6, we can work on the bounded subset $\Omega \times[0,1]$. Then, in virtue of Proposition 5.7, searching for an optimal pair $(\bar{u}, \bar{\sigma})$ amounts to find a saddle point for the bivariate functional

$$
\inf _{v \in \widehat{\mathcal{A}}} \sup _{\sigma \in \mathcal{K}} \int_{\Omega \times[0,1]} \sigma \cdot D v,
$$

with

$$
\begin{aligned}
& \widehat{\mathcal{A}}=\left\{v \in B V_{\infty}(\Omega \times \mathbb{R}): v=1 \text { for } t<0, v=0 \text { for } t>1, v=1 \text { on } \partial \Omega \times[0,1]\right\} \\
& \mathcal{K}=\left\{\sigma \in X_{1}(\Omega \times(0,1)): \sigma^{t}+\lambda \geq \frac{1}{2}\left|\sigma^{x}\right|^{2} \text { a.e. on } \Omega \times(0,1), \sigma^{t}(\cdot, 0) \geq 0 \text { a.e. on } \Omega\right\} .
\end{aligned}
$$

Notice carefully that the integration domain in $(6.4)$ is the product of $\Omega$ times the closed interval $[0,1]$. Actually, minimizing over $\widehat{\mathcal{A}}$ the functional $v \mapsto \int_{\Omega \times[0,1]} \sigma \cdot D v$ appearing in (6.4) is equivalent to minimizing over the space of functions $v \in B V(\Omega \times(0,1))$ satisfying the boundary condition $v=1$ on $\partial \Omega \times[0,1]$ the functional

$$
v \mapsto \int_{\Omega \times(0,1)} \sigma \cdot D v+\int_{\Omega}\left[\sigma^{t}(x, 0)\left(v\left(x, 0^{+}\right)-1\right)-\sigma^{t}(x, 1) v\left(x, 1^{-}\right)\right] d x
$$

being $v\left(x, 0^{+}\right)$and $v\left(x, 1^{-}\right)$respectively the traces of $v$ on $\Omega \times\{0\}$ and $\Omega \times\{1\}$.

Before proceeding to solve the min-max problem (6.4) let us recall that, if $(\bar{v}, \bar{\sigma})$ is an optimal pair, the function $\bar{v}$ should be a step function. Indeed, we expect that the primal problem (6.1) admits only one or at most a finite number of solutions. Then, by virtue of (4.14), the function $\bar{v}$ will take only the values 0 and 1 in case of a unique solution, or a finite number of values in $[0,1]$ in case of multiple solutions.

6.2. Numerical algorithms. In order to solve the saddle point problem (6.4), we adopt two different numerical schemes.

The first one is a primal-dual algorithm which generalizes a classical method of ArrowHurwicz [7], which we took from [34] (see also [35]). We choose an initial point $\left(v_{0}, \sigma_{0}\right) \in$ $\widehat{\mathcal{A}} \times \mathcal{K}$ and two positive time steps $\alpha, \beta$. Then, for each $n \in \mathbb{N}$, denoting by $h$ the size 
parameter of a cartesian grid in $\mathbb{R}^{N+1}$, we let

$$
\left\{\begin{array}{l}
\sigma_{n+1}^{h}=\Pi_{K}^{h}\left(\sigma_{n}^{h}+\alpha \nabla^{h} \bar{v}_{n}^{h}\right) \\
v_{n+1}^{h}=v_{n}^{h}+\beta \operatorname{div}^{h}\left(\sigma_{n+1}^{h}\right) \\
\bar{v}_{n+1}^{h}=2 v_{n+1}^{h}-v_{n}^{h},
\end{array}\right.
$$

where $\Pi_{K}^{h}$ is a suitable projection operator associated with the convex constraint $K(t)$. The convergence for system (6.5) requires that the stringent condition $\alpha \beta \leq c_{h}^{2} \leq 1$ is satisfied, where $c_{h}$ equals $2 \sqrt{N} / h$ (namely the norm of the discretized gradient operator). The computational cost in terms of the mesh size $h$ can be shown to be of order $\frac{1}{h^{N+2}}$. The second scheme is inspired from the projection method for Navier-Stokes system, in which a $L^{2}$-orthogonal projection is performed on the space of divergence free field (in this analogy, $\sigma$ and $v$ represent respectively the speed and the pressure of the fluid). Roughly, in our case we start from the reformulation of problem (6.4) as

$$
\inf _{p \in \mathcal{C}} \sup _{\sigma \in \mathcal{K}} \int_{\Omega \times[0,1]} \sigma \cdot p \quad \text { with } \mathcal{C}:=\{D v: v \in \widehat{\mathcal{A}}\} .
$$

Then we replace the second equation in $(6.5)$ by

$$
p_{n+1}^{h}=\Pi_{\mathcal{C}}^{h}\left(p_{n}^{h}-\beta \sigma_{n+1}^{h}\right),
$$

where $\Pi_{\mathcal{C}}^{h}$ is the $L^{2}$-orthogonal projector on the convex set $\mathcal{C}$. Denoting by $\left(\Delta^{h}\right)^{-1}$ the discretization of the inverse Dirichlet-Neumann Laplacian operator which associates to a function $\varphi$ the solution $w$ to

$$
\Delta w=\varphi, \quad w=0 \text { on } \partial \Omega \times(0,1), \quad \frac{\partial w}{\partial n}=0 \text { on } \Omega \times\{0,1\},
$$

we are led to the following semi-implicit algorithm

$$
\left\{\begin{array}{l}
\sigma_{n+1}^{h}=\Pi_{K}^{h}\left(\sigma_{n}^{h}+\alpha \nabla^{h} \bar{v}_{n}^{h}\right) \\
v_{n+1}^{h}=v_{n}^{h}-\beta\left(\Delta^{h}\right)^{-1}\left(\operatorname{div}^{h}\left(\sigma_{n+1}^{h}\right)\right) \\
\bar{v}_{n+1}^{h}=2 v_{n+1}^{h}-v_{n}^{h} .
\end{array}\right.
$$

Notice that 6.7) differs from (6.5) just in the term $-\left(\Delta^{h}\right)^{-1}\left(\operatorname{div}^{h}\left(\sigma_{n+1}^{h}\right)\right)$ which replaces $\operatorname{div}^{h}\left(\sigma_{n+1}^{h}\right)$.

The theoretical convergence of this second algorithm can be proved under the condition $\alpha \beta \leq 1$, which is independent from both the mesh side and the space dimension. Moreover, in this case the convergence occurs after a relatively small number of iterations. In fact, the inverse Laplacian computation is the most costly (in particular for $\Omega \subset \mathbb{R}^{2}$ when one works in $\mathbb{R}^{3}$ ), and the computational cost depends highly on the solver used for the inverse Laplace operator; if one uses a multigrid or a FFT solver, it can be of order $\frac{1}{h^{N+1} \log h}$.

6.3. Some simulations in case $N=1$. When the open set $\Omega$ is an interval $(0, a)$ of the real line, we can solve explicitly the primal problem, which reads

$$
\mathcal{I}(a, \lambda):=\inf \left\{\int_{0}^{a} \frac{\left|u^{\prime}\right|^{2}}{2}+\lambda|\{u \neq 0\}| d t: u \in W^{1,2}(0, a), u(0)=u(a)=1\right\} .
$$

The Euler-Lagrange equation written in the integrated conservation law form reads

$$
\frac{1}{2}\left|u^{\prime}\right|^{2}-\lambda \chi_{\{u \neq 0\}}=C .
$$


Two cases may occur, according to whether the measure of the level set $\{u=0\}$ is null or strictly positive. In the first case, the solution is the constant function equal to 1 on $(0, a)$, with cost equal to $\lambda a$. In the second case, the constant $C$ in 6.9 equals zero, so that $u^{\prime} \in\{0, \pm \sqrt{2 \lambda}\}$. Setting $E^{ \pm}=\left\{x \in(0, a): u^{\prime}= \pm \sqrt{2 \lambda}\right\}$, since $\int_{0}^{a} u^{\prime}=0$, there holds $\left|E^{+}\right|=\left|E^{-}\right|$, and the cost is $4 \lambda\left|E^{-}\right|$. On the other hand, since $u(0)=1$ and $u$ reaches the level zero, we have the lower bound $\left|E^{-}\right| \geq 1 / \sqrt{2 \lambda}$. Therefore, such a function $u$ can be a minimizer only if $a \geq 2 \sqrt{2 / \lambda}$, and in this case the minimal cost is larger than or equal to $2 \sqrt{2 \lambda}$, with equality if $E^{-}=(0,1 / \sqrt{2 \lambda}), E^{+}=(h-1 / \sqrt{2 \lambda}, a)$. To summarize, we have $\mathcal{I}(\lambda, a)=\min \{\lambda a, 2 \sqrt{2 \lambda}\}$, and

(i) for $a \in\left(0,2 \sqrt{\frac{2}{\lambda}}\right]$, the unique solution is $\bar{u}_{1} \equiv 1$;

(ii) for $a>2 \sqrt{\frac{2}{\lambda}}$, the unique solution is

$$
\bar{u}_{2}(x)= \begin{cases}-\sqrt{2 \lambda} x+1 & \text { if } x \in\left[0, \frac{1}{\sqrt{2 \lambda}}\right] \\ 0 & \text { if } x \in\left[\frac{1}{\sqrt{2 \lambda}}, a-\frac{1}{\sqrt{2 \lambda}}\right] \\ \sqrt{2 \lambda} x+1-\sqrt{2} a & \text { if } x \in\left[a-\frac{1}{\sqrt{2 \lambda}}, a\right]\end{cases}
$$

(iii) for $a=2 \sqrt{\frac{2}{\lambda}}$ there are two solutions, given by the two functions $\bar{u}_{1}$ and $\bar{u}_{2}$.

Contrarily to the primal problem, the dual problem does not admit easy explicit bounded solutions. In particular, the one obtained through the value function ( $c f$. Remark 3.9) blows up near the lateral boundary of the cylinder (see Remark 6.1 for more details).

Below we give some numerical results obtained, for $a=2$, by using the algorithm (6.7).

Figures 3, 4, 5 correspond to three cases $\lambda=1,2,4$. They represent the behaviour of the optimal $\bar{\sigma}$ and $\bar{v}$ in each case. Up to a translation of the interval $\Omega=(0,2)$ into $(-1,1)$, we can work on the cylinder $(-1,1) \times(0,1)$; then, for symmetry reasons, we limit ourselves to plot our functions on the right part $(0,1) \times(0,1)$ of the cylinder. Notice that the most important issue is the location of the discontinuity set of $\bar{v}$, as the free boundary is given by the intersection of this set with the horizontal axis.

For $\lambda=2$, we recover the two solutions $\bar{u}_{1}$ and $\bar{u}_{2}$ since the optimal function $\bar{v}$ exhibits three values (see Figure 4, were the regions in blue, red, and brown correspond respectively to the level sets $\{\bar{v}=0\},\{\bar{v}=0.8886\}$, and $\{\bar{v}=1\}$ ).

In constrast, for $\lambda=1$ or $\lambda=4$, when the primal problem admits a unique solution, the function $\bar{v}$ exhibits only two values (see the regions in blue and brown in Figures 3 and 5).

Remark 6.1. Let us compute the candidate calibration obtained for problem (6.8) through the method described in Remark 3.9. Through some straightforward computations it is easy to obtain that the value function introduced in 3.26 is given by

$$
\begin{aligned}
V(x, t) & =\inf \left\{\int_{0}^{x} f\left(u, u^{\prime}\right) d t: u \in W^{1,2}(0, h), u(0)=1, u(x)=t\right\} \\
& =\min \left\{\frac{1}{2} \frac{(t-1)^{2}}{x}+\lambda x, \sqrt{2 \lambda}(1+|t|)\right\}
\end{aligned}
$$



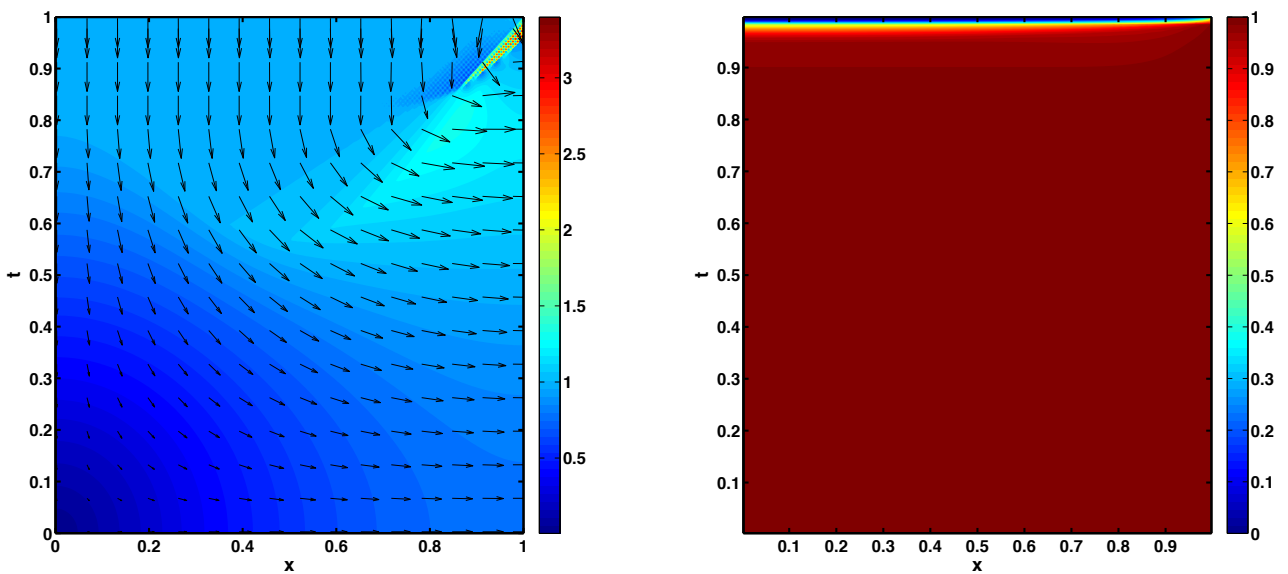

Figure 3. Streamlines of $\bar{\sigma}$ and level sets of $\bar{v}$ in the case $\lambda=1$
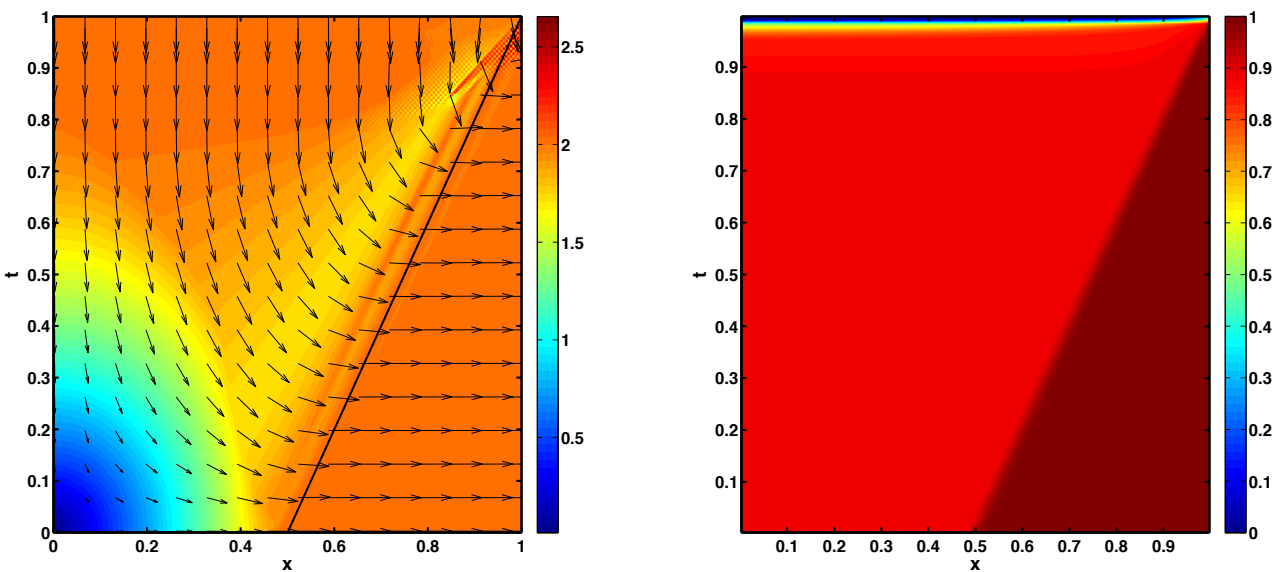

Figure 4. Streamlines of $\bar{\sigma}$ and level sets of $\bar{v}$ in the case $\lambda=2$
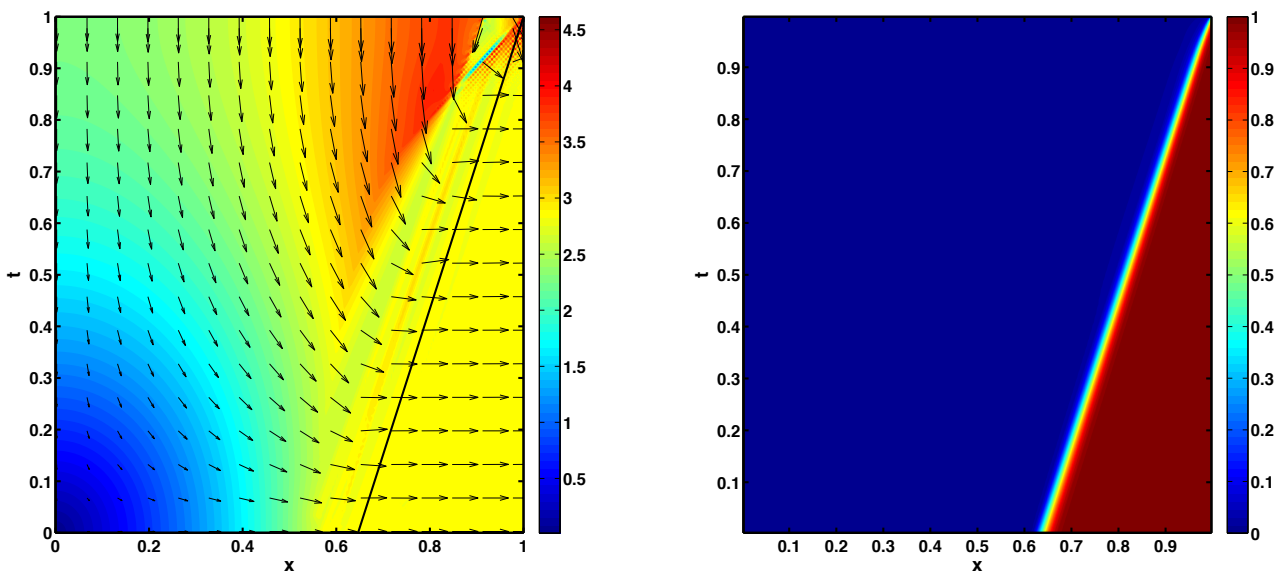

Figure 5. Streamlines of $\bar{\sigma}$ and level sets of $\bar{v}$ in the case $\lambda=4$ 


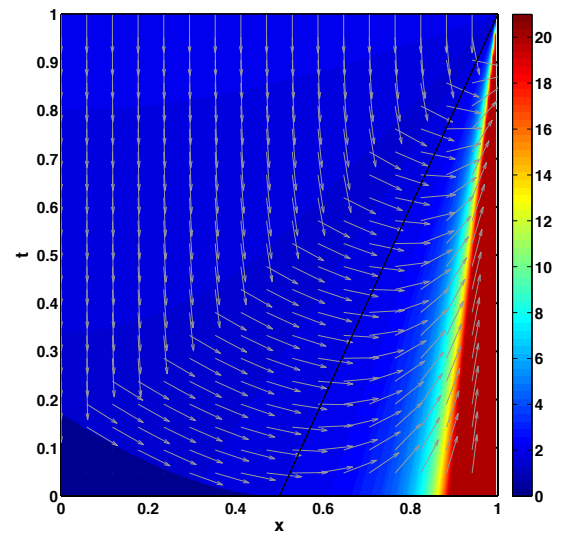

Figure 6 . Streamlines of the field $\widetilde{\sigma}$ given by the value function

Accordingly, the explicit expression of the vector field $\sigma(x, t):=\left(\partial_{t} V,-\partial_{x} V\right)$ reads

$$
\sigma(x, t)= \begin{cases}\left(\frac{t-1}{x}, \frac{1}{2} \frac{(t-1)^{2}}{x^{2}}-\lambda\right) & \text { if } x \leq \frac{1}{\sqrt{2 \lambda}}(1+\sqrt{t})^{2}, t>0, \text { or } x \leq \frac{1}{\sqrt{2 \lambda}}(1+|t|), t<0 \\ (\sqrt{2 \lambda}, 0) & \text { if } x>\frac{1}{\sqrt{2 \lambda}}(1+\sqrt{t})^{2}, t>0 \\ (-\sqrt{2 \lambda}, 0) & \text { if } x>\frac{1}{\sqrt{2 \lambda}}(1+|t|), t<0,\end{cases}
$$

It is easy to check that $\sigma$ satisfies conditions $(3.9)$ - (3.10). However, $V$ is not optimal for the formulation 3.25 of the dual problem because is not Lipschitz; indeed, it turns out that $\sigma$ blows up near $x=0$, see Figure 6 for a plot representing in case $\lambda=2$ the symmetrized field $\widetilde{\sigma}(x, t):=\left(\frac{1}{2}\left[\sigma^{x}(x, t)+\sigma^{x}(2-x, t)\right], \frac{1}{2}\left[\sigma^{t}(x, t)-\sigma^{t}(2-x, t)\right]\right.$ ) (which also satisfies conditions (3.9)-(3.10) ). Again, for symmetry reasons, the plot is restricted to the right half of the cylinder.

6.4. Some simulations in case $N=2$. By using the concavity of the map $\lambda \mapsto \mathcal{I}(\Omega, \lambda)$ one can check that, similarly to the one dimensional case, there exists a critical value $\lambda^{*}=\lambda^{*}(\Omega)$ below which the unique solution of the primal problem is $\bar{u}_{1} \equiv 1$, corresponding to the function $\bar{v}_{1} \in \widehat{\mathcal{A}}$ which vanishes identically in $\Omega \times(0,1)$. For $\lambda=\lambda^{*}(\Omega)$ this solution may coexist with a non constant solution $\bar{u}_{2}$, exhibiting a free boundary $E$.

Moreover, the function $\Omega \mapsto \lambda^{*}(\Omega)$ turns out to be monotone decreasing with respect to domain inclusions. In the special case when $\Omega=B_{R}:=\{|x|<R\}$, we find the explicit value $\lambda^{*}\left(B_{R}\right)=\frac{2 e}{R^{2}}$.

We now present some numerical simulations obtained for $\Omega=(-1,1)^{2}$. Noticing that $B_{1} \subset \Omega \subset B_{\sqrt{2}}$, we can predict a critical value $\lambda^{*}(\Omega)$ in the interval $(e, 2 e)$. In fact, by using the second algorithm described above with a mesh size $10^{-2}$ and by tuning the value of $\lambda$, we obtained the estimate $\lambda^{*}(\Omega) \sim 4.7$.

In Figures 7 and 8 we represent respectively the behaviour of the optimal field $\bar{\sigma}$ and of the optimal function $\bar{u}$ for $\lambda=2 e$ (for symmetry reasons, Figure 7 is referred just to a quarter of $\Omega$, namely to the set $\left.(0,1)^{2}\right)$. Notice that the free boundary is given by the frontier of the region in dark blue. 


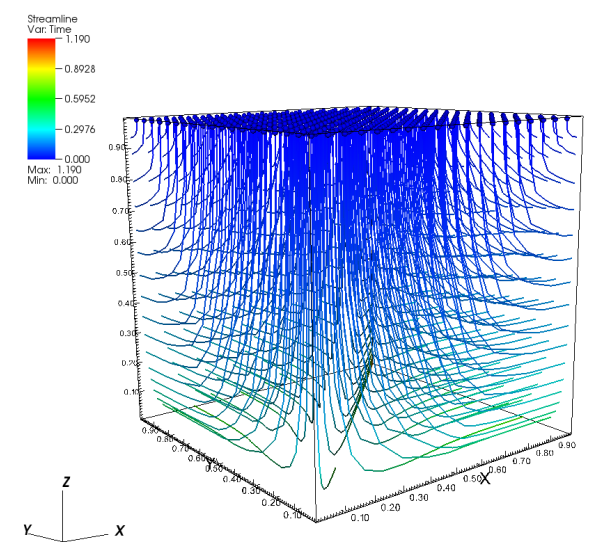

Figure 7. Streamlines of $\bar{\sigma}$ in the case $\lambda=2 e$
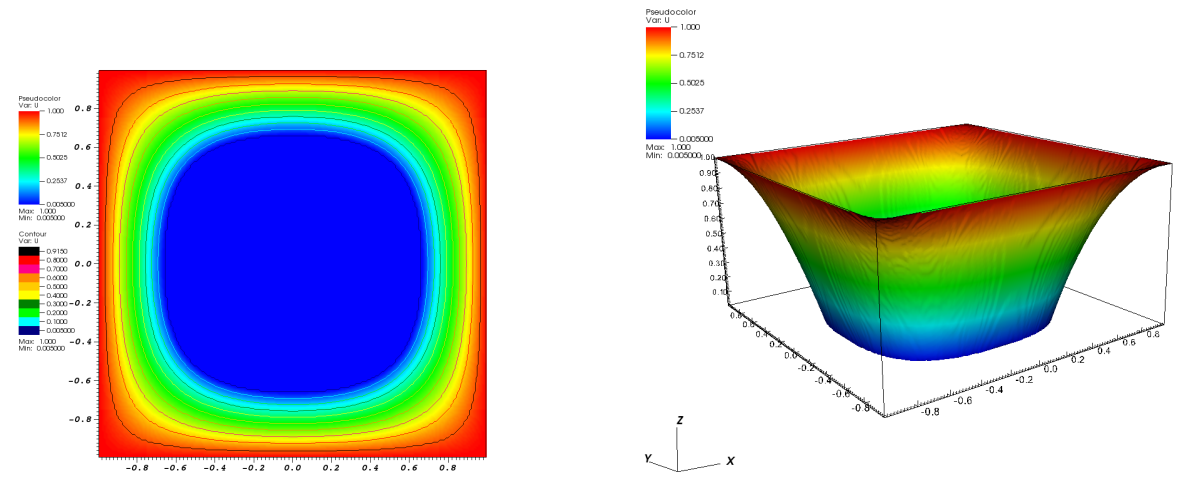

Figure 8. Level sets and plots of $\bar{u}$ in the case $\lambda=2 e$

\section{Completion of the Proofs}

In this section we prove the duality principle stated in Theorem 3.4 and the coarea formula stated in Theorem 4.10.

Before starting with the proof of Theorem 3.4 we give some preliminary lemmas.

Lemma 7.1. (i) If $\Gamma_{1} \neq \emptyset$, for every compact neighbourhood $U$ of $\Gamma_{1}$, there exists $\sigma_{U} \in \mathcal{B}$ such that

$$
\operatorname{spt}\left(\sigma_{U}\right) \subset U \times \mathbb{R}
$$

(ii) There exists $\sigma_{0} \in \mathcal{B}$ such that, for $\delta>0$ sufficiently small, it holds

$$
\|\eta\|_{L^{\infty}(\Omega \times \mathbb{R})} \leq \delta \Rightarrow \sigma_{0}+\eta \in \mathcal{K} .
$$


Proof. (i) Let $A:=U \cap \Omega$, that we can assume to be Lipschitz. Thanks to (3.8), we know there exists a field $q \in X_{1}(\Omega)$, with $\operatorname{spt}(q) \subseteq A$, such that

$$
\operatorname{div} q=\frac{\left|\Gamma_{1}\right|}{|A|} \chi_{A} \text { in } \Omega \quad q \cdot \nu_{A}=1 \text { on } \Gamma_{1} \quad q \cdot \nu_{A}=0 \text { on } \partial A \backslash \Gamma_{1} .
$$

We define the vector field $\sigma$ by

$$
\sigma(x, t)=\left(-\gamma^{\prime}(t) q(x), \frac{\left|\Gamma_{1}\right|}{|A|} \chi_{A}(x)\left[\gamma(t)-\inf _{\mathbb{R}} \gamma\right]+\lambda\right),
$$

being $q$ as in $(7.1)$, and $\lambda \in \mathbb{R}$ to be chosen later. By the choice of $q$, it is immediate that $\operatorname{div} \sigma=0$ in $\Omega \times \mathbb{R}$ and $\sigma \cdot \nu_{\Omega}=-\gamma^{\prime}$ on $\Gamma_{1} \times \mathbb{R}$. Let us show that it is possible to choose $\lambda$ such that $\sigma$ belongs to $\mathcal{K}$. By the growth condition from below in (2.6) satisfied by $f$, setting $k:=\sup _{\mathbb{R}} r$, it holds

$$
f_{z}^{*}\left(t, z^{*}\right) \leq b\left|z^{*}\right|^{p^{\prime}}+k, \quad \text { with } b=b(\alpha, p):=\frac{1}{p^{\prime}} \frac{1}{(\alpha p)^{p^{\prime}-1}},
$$

and $-f(t, 0) \leq r(t) \leq k$. Therefore, in order that $\sigma$ satisfies $(3.9)$ and $(3.10)$, it is enough to choose $\lambda$ such that

$$
\lambda \geq b\left\|\gamma^{\prime}\right\|_{\infty}^{p^{\prime}}\|q\|_{\infty}^{p^{\prime}}+k .
$$

(ii) Let us consider separately the cases $\Gamma_{1} \neq \emptyset$ and $\Gamma_{1}=\emptyset$.

Case $\Gamma_{1} \neq \emptyset$. We define the vector field $\sigma_{0}$ by

$$
\sigma_{0}(x, t):=\left(-\gamma^{\prime}(t) \psi(x), c_{\Omega} \gamma(t)+\lambda\right),
$$

being $\lambda \in \mathbb{R}$ to be chosen later, $c_{\Omega}:=|\partial \Omega| /|\Omega|$, and $\psi:=\nabla w$, with $w$ the unique solution to the boundary value problem $\Delta w=c_{\Omega}$ in $\Omega, w_{\nu}=1$ on $\partial \Omega$. Clearly $\sigma_{0} \in L^{\infty}\left(\Omega \times \mathbb{R} ; \mathbb{R}^{N+1}\right)$ and by construction it holds div $\sigma_{0}=0$ in $\Omega \times \mathbb{R}$ and $\left(\sigma_{0}\right)^{x} \cdot \nu_{\Omega}=-\gamma^{\prime}$ on $\partial \Omega \times \mathbb{R}$ (thus in particular on $\Gamma_{1} \times \mathbb{R}$ ). Let us check that it is possible to choose $\lambda$ so that $\sigma_{0}+\eta$ belongs to $\mathcal{K}$ if $\|\eta\|_{\infty} \leq \delta$.

We recall that, by our hypothesis $(2.9)$ (in its weaker version asked for $\Gamma_{0} \neq \emptyset$ ), and thanks to the boundedness of $r$, there exists a constant $m \in \mathbb{R}$ such that

$$
c_{\Omega} \gamma(t)-r(t) \geq m \quad \text { for } \mathcal{L}^{1} \text {-a.e. } t \in \mathbb{R} .
$$

In order that $\sigma_{0}+\eta$ satisfies $(3.9)$, we need to choose $\lambda$ such that

$$
|q|<\delta \Rightarrow c_{\Omega} \gamma(t)+\lambda+q^{t} \geq f_{z}^{*}\left(t,-\gamma^{\prime}(t) \psi(x)+q^{x}\right) \quad \text { for } \mathcal{L}^{N+1} \text {-a.e. }(x, t) \in \Omega \times \mathbb{R} .
$$

Since, by the growth condition from below in 2.6 satisfied by $f$, it holds $f_{z}^{*}\left(t, z^{*}\right) \leq$ $b\left|z^{*}\right|^{p^{\prime}}+r(t)$, it is enough to have

$|q|<\delta \Rightarrow c_{\Omega} \gamma(t)+\lambda+q^{t} \geq b\left|-\gamma^{\prime}(t) \psi(x)+q^{x}\right|^{p^{\prime}}+r(t) \quad$ for $\mathcal{L}^{N+1}$-a.e. $(x, t) \in \Omega \times \mathbb{R}$.

In turn, in view of 7.2 , we are reduced to choose $\lambda$ so that

$$
|q|<\delta \Rightarrow m+\lambda \geq b\left|-\gamma^{\prime}(t) \psi(x)+q^{x}\right|^{p^{\prime}}-q^{t} \quad \text { for } \mathcal{L}^{N+1} \text {-a.e. }(x, t) \in \Omega \times \mathbb{R},
$$

which is clearly possible since $\psi$ is bounded and $\gamma$ is Lipschitz.

In order that $\sigma_{0}+\eta$ satisfies (3.10), we need to choose $\lambda$ such that

$$
|q|<\delta \Rightarrow c_{\Omega} \gamma(t)+\lambda+q^{t} \geq-f(t, 0) \quad \forall t \in D, \text { for } \mathcal{L}^{N} \text {-a.e. } x \in \Omega .
$$

This is possible because, by the growth assumption (2.6), we have $-f(t, 0) \leq r(t)$, and hence, in view of $(7.2)$, it is enough to choose $\lambda$ so that

$$
|q|<\delta \Rightarrow m+\lambda \geq-q^{t} .
$$


Case $\Gamma_{1}=\emptyset$. We define the vector field $\sigma_{0}$ simply by

$$
\sigma_{0}(x, t):=(0, \lambda) .
$$

Clearly, it holds $\sigma_{0} \in L^{\infty}\left(\Omega \times \mathbb{R} ; \mathbb{R}^{N+1}\right)$ and $\operatorname{div} \sigma_{0}=0$ in $\Omega \times \mathbb{R}$. We have just to choose $\lambda$ so that $\sigma_{0}+\eta$ belongs to $\mathcal{K}$ if $\|\eta\|_{\infty} \leq \delta$.

In order that $\sigma_{0}+\eta$ satisfies 3.9$)$, in view of the inequality $f_{z}^{*}\left(t, z^{*}\right) \leq b\left|z^{*}\right|^{p^{\prime}}+r(t)$, it is enough to have

$$
|q|<\delta \Rightarrow \lambda+q^{t} \geq b\left|q^{x}\right|^{p^{\prime}}+r(t) \quad \text { for } \mathcal{L}^{N+1} \text {-a.e. }(x, t) \in \Omega \times \mathbb{R} .
$$

This is clearly possible since we assumed $r(t)$ bounded.

In order that $\sigma_{0}+\eta$ satisfies 3.10 , in view of the inequality $-f(t, 0) \leq r(t)$, it is enough to choose $\lambda$ such that

$$
|q|<\delta \Rightarrow \lambda+q^{t} \geq r(t) \quad \forall t \in D, \text { for } \mathcal{L}^{N} \text {-a.e. } x \in \Omega .
$$

Once again, this is possible thanks to the boundedness of $r$.

Lemma 7.2. For every $\sigma \in X_{1}(\Omega \times \mathbb{R})$ and every $v \in B V_{\infty}(\Omega \times \mathbb{R})$, it holds

$$
H(v)=\sup \left\{\int_{\Omega \times \mathbb{R}}(\sigma+\eta) \cdot D v: \eta \in \mathcal{D}\left(\Omega \times \mathbb{R} ; \mathbb{R}^{N+1}\right), \sigma+\eta \in \mathcal{K}\right\} .
$$

Proof. Let $G(\sigma)$ denote the right hand side of $(7.3)$. The map $\rho: t \in \mathbb{R} \mapsto G(t \sigma)$ is convex (as it is the supremum of affine functions). By Lemma 4.4, it holds $\rho(0)=H(v)$, whereas $\rho(t) \leq H(v)$ for every $t$ by Lemma 4.6. It follows that $\rho(t)$ is constant. We deduce in particular that $G(\sigma)=\rho(1)=\rho(0)=H(v)$.

\section{- Proof of Theorem 3.4.}

Thanks to the equality (4.13) established in Theorem 4.1, the thesis of Theorem 3.4 (namely the equality $\mathcal{I}^{*}=\mathcal{I}$ ) can be reformulated as

$$
\mathcal{I}^{*}=\inf \{\widehat{E}(v): v \in \widehat{\mathcal{A}}\} .
$$

In order to prove $(7.4)$, we introduce on $\mathcal{C}_{0}\left(\Omega \times \mathbb{R} ; \mathbb{R}^{N+1}\right)$ the perturbation function

$$
\begin{gathered}
\Phi(\eta):=\inf \left\{-\int_{G_{u_{0}}} \sigma \cdot \nu_{u_{0}} d \mathcal{H}^{N}-\int_{\Gamma_{1}} \gamma\left(u_{0}\right) d \mathcal{H}^{N-1}: \sigma \in X_{1}\left(\Omega \times \mathbb{R} ; \mathbb{R}^{N+1}\right),\right. \\
\left.\operatorname{div} \sigma=0, \sigma^{x} \cdot \nu_{\Omega}=-\gamma^{\prime} \text { on } \Gamma_{1} \times \mathbb{R}, \sigma+\eta \in \mathcal{K}\right\} .
\end{gathered}
$$

It is easy to check that the map $\eta \mapsto \Phi(\eta)$ is convex. Moreover, in view of the choice of admissible fields $\sigma$ in the definition of $\Phi(\eta)$, it holds

$$
\mathcal{I}^{*}=-\Phi(0) .
$$

Let us compute $\Phi(0)$. Observe that $\Phi$ is continuous at 0 : namely, for any $\eta$ with $\|\eta\|_{\infty} \leq \delta$, thanks to Lemma 7.1 (ii) it holds

$$
\Phi(\eta) \leq-\int_{G_{u_{0}}} \sigma_{0} \cdot \nu_{u_{0}} d \mathcal{H}^{N}-\int_{\Gamma_{1}} \gamma\left(u_{0}\right) d \mathcal{H}^{N-1}
$$

Hence we have

$$
-\Phi(0)=-\Phi^{* *}(0)=\min \left(\Phi^{*}\right)
$$


where $\Phi^{*}=\Phi^{*}(\lambda)$ denotes the Fenchel conjugate of $\Phi$ in the duality between continuous functions and bounded measures. Let us compute $\Phi^{*}$, and let us show that it satisfies

$$
\Phi^{*}(\lambda)= \begin{cases}\widehat{E}(v) & \text { if } \lambda=D v, \text { with } v \in \widehat{\mathcal{A}} \\ +\infty & \text { otherwise. }\end{cases}
$$

Once proved (7.7), our proof will be achieved. Indeed, (7.7) implies in particular that $\min \left(\Phi^{*}\right)=\min \{\widehat{E}(v): v \in \widehat{\mathcal{A}}\}$. Taking into account (7.5) and (7.6), we deduce that the required equality $(7.4)$ is satisfied.

In order to establish (7.7), we fix now a bounded vector measure $\lambda$ such that $\Phi^{*}(\lambda)<+\infty$ and we proceed in three steps.

Step 1. If $\Gamma_{1} \neq \emptyset$, for every compact neighborhood $U$ of $\Gamma_{1}$ and every bounded continuous $\psi: \bar{\Omega} \times \mathbb{R} \rightarrow \mathbb{R}^{N+1}$, it holds

$$
\begin{gathered}
\left\langle\lambda-D \mathbb{1}_{u_{0}}, \psi\right\rangle=0 \text { whenever } \operatorname{div} \psi=0 \text { in } \Omega \times \mathbb{R} \text { and } \psi=0 \text { on } U \times \mathbb{R} \\
\int_{(\Omega \backslash U) \times \mathbb{R}} h_{f}(t, \lambda)<+\infty .
\end{gathered}
$$

If $\Gamma_{1}=\emptyset$, conditions (7.8) and (7.9) hold true with $U=\emptyset$.

Assume first that $\Gamma_{1} \neq \emptyset$. Given a compact neighborhood $U$ of $\Gamma_{1}$ and a function $\psi$ as in (7.8), we consider the vector field $\sigma=\sigma_{U}+\psi$ with $\sigma_{U}$ chosen according to Lemma 7.1 (i). Since such $\sigma$ is divergence free and satisfies $\sigma^{x} \cdot \nu_{\Omega}=-\gamma^{\prime}$ on $\Gamma_{1} \times \mathbb{R}$, in view of the definition of $\Phi$, one has:

$$
\Phi(\eta) \leq-\int_{G_{u_{0}}}\left(\sigma_{U}+\psi\right) \cdot \nu_{u_{0}} d \mathcal{H}^{N}-\int_{\Gamma_{1}} \gamma\left(u_{0}\right) d \mathcal{H}^{N-1}
$$

for every smooth field $\eta$ with compact support in $\Omega \backslash U$ such that $\psi+\eta \in \mathcal{K}$.

This implies

$$
\Phi^{*}(\lambda) \geq\langle\lambda, \eta+\psi\rangle+\left\langle D \mathbb{1}_{u_{0}}-\lambda, \psi\right\rangle+\int_{G_{u_{0}}} \sigma_{U} \cdot \nu_{u_{0}} d \mathcal{H}^{N}+\int_{\Gamma_{1}} \gamma\left(u_{0}\right) d \mathcal{H}^{N-1},
$$

where we have used the identity $\int_{G_{u_{0}}} \psi \cdot \nu_{u_{0}}=\left\langle D \mathbb{1}_{u_{0}}, \psi\right\rangle$. Now by fixing $\psi$ and taking the supremum with respect to $\eta$ satisfying the conditions above, by exploiting Lemma 7.2 applied on $\Omega \backslash U$, we deduce that, for a suitable constant $C$, there holds:

$$
\Phi^{*}(\lambda) \geq \int_{(\Omega \backslash U) \times \mathbb{R}} h_{f}(t, \lambda)+\left\langle D \mathbb{1}_{u_{0}}-\lambda, \psi\right\rangle+C .
$$

Thus, since by assumption $\Phi^{*}(\lambda)$ is finite, $(7.8)$ and $(7.9)$ follow.

In case $\Gamma_{1}=\emptyset$, we can repeat the same proof above with $\sigma_{U} \equiv 0$.

Step 2. There exists a scalar function $v \in L_{\text {loc }}^{1}(\Omega \times \mathbb{R})$, with $v(x, \cdot)$ monotone nonincreasing, such that $\lambda=D v$. Moreover, up to adding a constant to $v$, we have $v \in \widehat{\mathcal{A}}$, as it holds:

$$
\begin{gathered}
v \in B V_{\infty}(\Omega \times \mathbb{R} ;[0,1]), \quad v(x,-\infty)=1, \quad v(x, \infty)=0 \\
v-v_{0} \in L^{1}(\Omega \times \mathbb{R}) \\
v=\mathbb{1}_{u_{0}} \text { on } \Gamma_{0} \times \mathbb{R} .
\end{gathered}
$$


From $(7.8)$, since $U$ is arbitrarily small (and empty in case $\Gamma_{1}=\emptyset$ ), we infer that the bounded measure $\lambda-D \mathbb{1}_{u_{0}}$ is orthogonal to all smooth vector fields $\psi$ which are divergence free and compactly supported in $\Omega \times \mathbb{R}$. As $\Omega \times \mathbb{R}$ is simply connected, this implies the existence of a scalar function $v \in L_{\text {loc }}^{1}(\Omega \times \mathbb{R})$ such that $\lambda=D v$. Then, since $\int_{K \times \mathbb{R}} h_{f}(t, \lambda)<+\infty$ for every compact set $K \subset \Omega$, we infer that $-D_{t} v$ is a non-negative measure on $\Omega \times \mathbb{R}$, which yields the desired monotonicity property of $v(x, \cdot)$ for a.e. $x \in \Omega$. Let us now prove that $v$ satisfies (7.10, 7 7.11), and (7.12).

To prove $(7.10)$, we choose $\varphi \in \mathcal{D}\left(\Omega ; \mathbb{R}^{+}\right)$and we set $\psi=(0, \varphi(x))$. Integrating by parts over $\Omega \times(-R,+R)$ and taking into acount that for a.e. $x \in \Omega, v(x,-R+0)-v(x, R-0)$ is non negative and converges increasingly to $\operatorname{var}(v(x, \cdot))$ as $R \rightarrow+\infty$, we obtain

$$
\begin{aligned}
\left\langle D v-D v_{0}, \psi\right\rangle & =\lim _{R \rightarrow+\infty} \int_{\Omega} \varphi(x)[v(x, R-0)-(v(x,-R+0)-1)] d x \\
& =\int_{\Omega} \varphi(x)(1-\operatorname{var}(v(x, \cdot)) d x .
\end{aligned}
$$

By the arbitrariness of $\varphi$, if we combine the above equality with $(7.8)$ and with the identity $\left\langle D \mathbb{1}_{u_{0}}-D v_{0}, \psi\right\rangle=0$, we get

$$
\operatorname{var}(v(x, \cdot))=1 \quad \text { for a.e. } x \in \Omega \text {. }
$$

Next, we consider a function $\varphi \in \mathcal{D}(\Omega)$ such that $\int_{\Omega} \varphi d x=0$, to which we associate a vector field $q \in L^{\infty}\left(\Omega ; \mathbb{R}^{N}\right)$ such that $-\operatorname{div}_{x} q=\varphi$ in $\Omega$ and $q \cdot \nu_{\Omega}=0$ on $\partial \Omega$. Set:

$$
\psi(x, t):=\left(\alpha^{\prime}(t) q(x), \alpha(t) \varphi(x)\right), \quad \text { with } \alpha(t):=H(t)\left(1-e^{-t}\right)
$$

(being $H$ the Heavyside function). Then, integrating once more by parts over $\Omega \times(-R,+R)$ and letting $R$ tend to $+\infty$, we obtain

$$
\left\langle D v-D v_{0}, \psi\right\rangle=\lim _{R \rightarrow+\infty} \int_{\Omega} \varphi(x) \alpha(R) v(x, R-0) d x=\int_{\Omega} \varphi(x) v(x,+\infty) d x,
$$

where in the second equality we use dominated convergence taking into account that $|v(\cdot, R-0)| \leq 1+\left|v\left(\cdot, t_{0}\right)\right|$ for a suitable $t_{0}>0$ such that $v\left(\cdot, t_{0}\right)=v\left(\cdot, t_{0} \pm 0\right)$ belongs to $L^{1}(\Omega)$. Then, by applying $(7.8)$ to the function $\psi$ introduced in (7.14), and recalling the arbitrariness of the smooth function $\varphi$ with vanishing average, we deduce that $v(x,+\infty)$ is a constant that we may fix to be zero. Thus, with the help of (7.13), we conclude the proof of 7.10$)$.

To prove (7.11), we fix $\sigma_{0} \in \mathcal{B}$ (for instance, we can take the one given by Lemma 7.1 (ii)). Similarly as above, we integrate by parts over $\Omega \times(-R \times R)$, and we obtain:

$$
\begin{aligned}
\left\langle D v-D v_{0}, \sigma_{0}\right\rangle=\lim _{R \rightarrow+\infty} & \left(\int _ { \Omega } \left[\sigma_{0}^{t}(x, R) v(x, R-0)+\sigma_{0}^{t}(x,-R)(1-v(x,-R+0)] d x\right.\right. \\
& \left.-\int_{\Gamma_{1} \times(-R, R)} \gamma^{\prime}(t)\left(v-v_{0}\right) d \mathcal{H}^{N-1}(x) d t\right) \\
=- & \lim _{R \rightarrow+\infty}\left(\int_{\Gamma_{1} \times[0, R)} \gamma^{\prime}(t) v d \mathcal{H}^{N-1}(x) d t\right. \\
- & \left.\int_{\left.\Gamma_{1} \times(-R, 0)\right)} \gamma^{\prime}(t)(1-v) d \mathcal{H}^{N-1}(x) d t\right)
\end{aligned}
$$

where in the second equality we used the fact that $\sigma_{0}^{t}$ is bounded together with the convergence of $v(\cdot, R-0)$ and of $1-v(\cdot,-R+0)$ to 0 in $L^{1}(\Omega)$. 
Now, recalling that $u_{s}$ are defined as in (4.6), and using the slicing property (4.36) proved in Proposition 4.13, we can rewrite the above equality as

$$
\begin{aligned}
&\left\langle D v-D v_{0}, \sigma_{0}\right\rangle=-\lim _{R \rightarrow+\infty} \int_{0}^{1} d s\left(\int_{\Gamma_{1} \cap\left\{u_{s} \geq 0\right\}} \gamma\left(u_{s} \wedge R\right) d \mathcal{H}^{N-1}(x)\right. \\
&\left.\quad+\int_{\Gamma_{1} \cap\left\{u_{s}<0\right\}} \gamma\left(u_{s} \vee-R\right) d \mathcal{H}^{N-1}(x)\right) \\
&=-\lim _{R \rightarrow+\infty} \int_{0}^{1} d s \int_{\Gamma_{1}} \gamma\left(u_{s}^{R}\right) d \mathcal{H}^{N-1}(x)
\end{aligned}
$$

where $u_{s}^{R}:=\left(u_{s} \wedge R\right) \vee-R$. Clearly $u_{s}^{R} \rightarrow u_{s}$ as $R \rightarrow+\infty$. Then, since $\gamma$ is assumed to be bounded from below, by applying Fatou's Lemma we get

$$
\left\langle D v_{0}-D v, \sigma_{0}\right\rangle=\liminf _{R \rightarrow+\infty} \int_{0}^{1} d s \int_{\Gamma_{1}} \gamma\left(u_{s}^{R}\right) d \mathcal{H}^{N-1}(x) \geq \int_{0}^{1} d s \int_{\Gamma_{1}} \gamma\left(u_{s}\right) d \mathcal{H}^{N-1}(x) .
$$

Recalling that $\left\langle D v-D v_{0}, \sigma_{0}\right\rangle$ is finite, we infer that

$$
\int_{0}^{1} d s \int_{\Gamma_{1}} \gamma\left(u_{s}\right) d \mathcal{H}^{N-1}(x)<+\infty .
$$

Now, by Proposition 4.12 and Step 1, we know that

$$
\int_{0}^{1} d s \int_{\Omega} f\left(u_{s}, \nabla u_{s}\right) d x=\int_{\Omega \times \mathbb{R}} h_{f}(t, D v)<+\infty .
$$

Notice in particular that, in case $\Gamma_{1}=\emptyset$, the last inequality follows from 7.9 , applied with $U=\emptyset$. In case $\Gamma_{1}=\emptyset$, we can still apply $(7.9)$ by letting $\Omega \backslash U$ increase to $\Omega$; this is possible thanks to the fact that $h_{f}(t, \lambda)$ is bounded below by a multiple of the total variation of $\lambda$. The last assertion is easily checked, since, for all $(t, q)$ with $q \neq 0$ such that $h_{f}(t, q)<+\infty$, it holds

$$
h_{f}(t, q)=-q^{t} f\left(t,-\frac{q^{x}}{q^{t}}\right) \geq-q^{t}\left|\frac{q^{x}}{q^{t}}\right|^{p}+q^{t} r(t) \geq q^{t} r(t),
$$

and our assumption (2.7) ensures that $r(t)$ is bounded.

Combining (7.15) and (7.16), we deduce that $\int_{0}^{1} E\left(u_{s}\right) d s<+\infty$. In view of the estimate (2.10) obtained in the proof of Proposition 2.2. we deduce that $\int_{0}^{1}\left\|u_{s}\right\|_{W^{1, p}(\Omega)} d s<+\infty$. This implies (7.11) since $\int_{\Omega \times \mathbb{R}}\left|v-v_{0}\right| d x d t=\int_{0}^{1} d s \int_{\Omega}\left|u_{s}\right| d x$.

To conclude the proof of Step 2, it remains to show (7.12). To that aim, it is enough to apply (7.8). Indeed integrating by parts we obtain $\int_{\Gamma_{0} \times \mathbb{R}}\left(v-\mathbb{1}_{u_{0}}\right) \psi \cdot \nu_{\Omega}=0$ for every bounded continuous function $\psi$ as in (7.8), and the conclusion follows recalling (3.8).

Step 3. There holds $\Phi^{*}(\lambda)=\widehat{E}(v)$.

Let $\sigma \in X_{1}\left(\Omega \times \mathbb{R} ; \mathbb{R}^{N+1}\right)$. We observe that, by Step 2 , the duality bracket $\sigma \cdot D v$ is well defined (cf. 4.2) ). Moreover, by Lemma 7.2 it holds

$$
H(v)=\sup \left\{\int_{\Omega \times \mathbb{R}}(\sigma+\eta) \cdot D v: \eta \in \mathcal{D}\left(\Omega \times \mathbb{R} ; \mathbb{R}^{N+1}\right), \sigma+\eta \in \mathcal{K}\right\} .
$$


We are now ready to compute the Fenchel conjugate of $\Phi$. We have :

$$
\begin{aligned}
\Phi^{*}(\lambda)= & \sup \left\{\int_{\Omega \times \mathbb{R}} \eta \cdot D v-\Phi(\eta): \eta \in \mathcal{C}_{0}\left(\Omega \times \mathbb{R} ; \mathbb{R}^{N+1}\right)\right\} \\
= & \sup \left\{\int_{\Omega \times \mathbb{R}} \eta \cdot D v-\Phi(\eta): \eta \in \mathcal{D}\left(\Omega \times \mathbb{R} ; \mathbb{R}^{N+1}\right)\right\} \\
= & \sup \left\{\int_{\Omega \times \mathbb{R}}(\eta+\sigma) \cdot D v+\int_{G_{u_{0}}} \sigma \cdot \nu_{u_{0}} d \mathcal{H}^{N}+\int_{\Gamma_{1}} \gamma\left(u_{0}\right) d \mathcal{H}^{N-1}-\langle D v, \sigma\rangle:\right. \\
& \eta \in \mathcal{D}\left(\Omega \times \mathbb{R} ; \mathbb{R}^{N+1}\right), \sigma \in X_{1}\left(\Omega \times \mathbb{R} ; \mathbb{R}^{N+1}\right), \\
& \left.\operatorname{div} \sigma=0, \sigma^{x} \cdot \nu_{\Omega}=-\gamma^{\prime} \text { on } \Gamma_{1} \times \mathbb{R}, \sigma+\eta \in \mathcal{K}\right\} \\
= & \int_{\Omega \times \mathbb{R}} h_{f}(t, D v)+\int_{\Gamma_{1}} \gamma\left(u_{0}\right) d \mathcal{H}^{N-1}+\sup \left\{\left\langle D \mathbb{1}_{u_{0}}-D v, \sigma\right\rangle:\right. \\
& \left.\sigma \in X_{1}\left(\Omega \times \mathbb{R} ; \mathbb{R}^{N+1}\right), \operatorname{div} \sigma=0, \sigma^{x} \cdot \nu_{\Omega}=-\gamma^{\prime} \text { on } \Gamma_{1} \times \mathbb{R}\right\},
\end{aligned}
$$

where :

- the first equality is just the definition of $\Phi^{*}$;

- the second equality follows from the density of $\mathcal{D}\left(\Omega \times \mathbb{R} ; \mathbb{R}^{N+1}\right)$ in $\mathcal{C}_{0}\left(\Omega \times \mathbb{R} ; \mathbb{R}^{N+1}\right)$ and from the continuity of the convex function $\Phi$ at 0 ;

- the third equality is just the definition of $\Phi$;

- the fourth equality holds by Lemma 7.2 .

Finally we observe that, thanks to 4.40 , the expression of $\Phi^{*}(\lambda)$ appearing in the fourth equality above coincides with

$$
\int_{\Omega \times \mathbb{R}} h_{f}(t, D v)+\int_{\Gamma_{1} \times \mathbb{R}}\left(v-v_{0}\right) \gamma^{\prime}(t) d \mathcal{H}^{N}=\widehat{E}(v) .
$$

Since from Step 2 we already know that $v \in \widehat{\mathcal{A}}$, the proof of $(7.7)$ is complete.

\section{- Proof of Theorem 4.10}

Throughout the proof we set for brevity

$$
u_{t}(x):=\chi_{\{u>t\}}(x) .
$$

Let us first show that the map $t \mapsto J\left(u_{t}\right)$ is Lebesgue measurable.

For every fixed open set $V \subset \subset A$, consider the function of a real variable defined by

$$
\psi_{V}(t):=\int_{V} u_{t}(x) d x .
$$

Clearly $\psi_{V}$ is monotone decreasing, non negative and bounded; in particular, it turns out to be continuous on $\mathbb{R} \backslash D_{V}$, where $D_{V}$ is a countable subset of $\mathbb{R}$ (depending on $V$ ). Moreover, since

$$
\forall t, \quad \forall \delta>0, \quad \int_{V}\left|u_{t}-u_{t+\delta}\right| d x=\psi_{V}(t)-\psi_{V}(t+\delta),
$$

the map $t \mapsto u_{t}$ is continuous from $\mathbb{R} \backslash D_{V}$ to $L^{1}(V)$. Then, by considering an increasing sequence of open sets $V_{h} \uparrow A$, and exploiting the assumption that $J$ is lower semicontinuous on $L_{\text {loc }}^{1}(A)$, we obtain that the map $t \mapsto J\left(u_{t}\right)$ is lower semicontinuous on $\mathbb{R} \backslash D$, with $D=\cup_{h} D_{V_{h}}$ countable. Consequently, the map $t \mapsto J\left(u_{t}\right)$ is Lebesgue-measurable on $\mathbb{R}$.

We now prove separately the inequality $J(u) \leq \int_{\mathbb{R}} J\left(u_{t}\right) d t$ and its converse. 
- Proof of the inequality $J(u) \leq \int_{\mathbb{R}} J\left(u_{t}\right) d t$

Since $J$ is convex, lower semicontinuous, and proper (recall that by assumption $J\left(\chi_{A}\right)=0$ ), we have $J^{* *}=J$, where $J^{* *}$ is the Fenchel biconjugate in the duality between $L_{\text {loc }}^{1}(A)$ and the space $L_{c}^{\infty}(A)$ of bounded functions with compact support. Namely,

$$
J(u)=J^{* *}(u)=\sup \left\{\int_{A} u w d x-J^{*}(w): w \in L_{c}^{\infty}(A)\right\} .
$$

Let us compute $J^{*}$. We claim that

$$
J^{*}(w)= \begin{cases}0 & \text { if } w \in X \\ +\infty & \text { otherwise }\end{cases}
$$

for some nonempty closed convex set $X \subseteq\left\{w \in L_{c}^{\infty}(A): \int_{A} w d x=0\right\}$

We begin by showing that $J^{*}$ takes only the values 0 and $+\infty$. By definition, there holds

$$
J^{*}(w)=\sup \left\{\int_{A} u w d x-J(u): u \in L_{\mathrm{loc}}^{1}(A)\right\} \quad \forall w \in L_{c}^{\infty}(A) .
$$

Let $w \in L_{c}^{\infty}(A)$ be fixed. If $J^{*}(w) \neq 0$, necessarily there exists some $u \in L_{\text {loc }}^{1}(A)$ such that $\int_{A} u w d x-J(u)=: r \neq 0$. Since for every $\lambda \geq 0$ we have $\int_{A}(\lambda u) w d x-J(\lambda u)=\lambda r$, we infer that $J^{*}(w)=+\infty$ if $r$ is positive (by letting $\lambda$ tend to $+\infty$ ) and $J^{*}(w) \geq 0$ if $r$ is negative (by letting $\lambda$ tend to 0 ); moreover, we see that that $J^{*}(w)$ cannot be strictly positive unless it is $+\infty$ (because if $J^{*}(w)>0$ there exists some $u \in L_{\text {loc }}^{1}(A)$ such that $\int_{A} u w d x-J(u)>0$, and arguing as above we see that $\left.J^{*}(w)=+\infty\right)$. We deduce that $J^{*}$ is of the form $(7.18)$ for some subset $X$ of $L_{c}^{\infty}(A)$. Since $J^{*}$ is convex, lower semicontinuous, and proper, $X$ is a nonempty closed convex subset of $L_{c}^{\infty}(A)$. Moreover, if $w \in X$, taking into account that by assumption $J\left(\chi_{A}\right)=0$, we have

$$
0=J^{*}(w) \geq \sup _{\lambda \in \mathbb{R}}\left[\lambda \int_{A} w d x\right]
$$

hence all functions in $X$ have zero mean on $A$, which concludes the proof of the claim.

We infer from (7.17) and 7.18 that

$$
J(u)=\sup _{w \in X} \int_{A} u w d x .
$$

As a next step let us show that, for every $w \in X$, setting $j_{w}(t):=\int_{A} u_{t} w d x$, there holds

$$
\int_{A} u w d x=\int_{-\infty}^{\infty} j_{w}(t) d t
$$

To that aim, we apply Fubini's theorem to compute the following two integrals:

$$
\begin{aligned}
\int_{u \geq 0} u w d x & =\int_{u \geq 0} \int_{0}^{u(x)} w(x) d t d x=\int_{u \geq 0} w(x) \int_{0}^{u(x)} d t d x \\
& =\int_{u \geq 0} w(x) \int_{0}^{+\infty} u_{t}(x) d t d x=\int_{0}^{+\infty} \int_{A} u_{t} w d x d t=\int_{0}^{+\infty} j_{w}(t) d t
\end{aligned}
$$


and

$$
\begin{aligned}
\int_{u \leq 0} u w d x & =-\int_{u \leq 0} w \int_{u(x)}^{0} d t d x=-\int_{u \leq 0} w \int_{-\infty}^{0}\left(1-u_{t}(x)\right) d t d x \\
& =\int_{-\infty}^{0} \int_{u \leq 0}\left(u_{t} w-w\right) d x d t=\int_{-\infty}^{0}\left[\int_{u \leq 0} u_{t} w d x+\int_{u>0} w d x\right] d t \\
& =\int_{-\infty}^{0}\left[\int_{u \leq 0} u_{t} w d x+\int_{u>0} u_{t} w d x\right] d t=\int_{-\infty}^{0} j_{w}(t) d t .
\end{aligned}
$$

Notice that, in the computation of the second integral (fourth equality), we used the fact that $w$ has zero mean on $A$.

By 7.19 and 7.20 , we have

$$
J(u)=\sup _{w \in X} \int_{-\infty}^{\infty} j_{w}(t) d t \leq \int_{-\infty}^{\infty} \mathcal{L}^{1}-\underset{w \in X}{\operatorname{ess} \sup }\left(j_{w}\right) d t
$$

Since we know from the first part of the proof that the map $t \mapsto u_{t}$ is continuous from $\mathbb{R} \backslash D$ to $L_{\text {loc }}^{1}(A)$ (with $D$ countable), taking into account that $w \in L_{c}^{\infty}(A)$ we see that $j_{w}(t)$ is continuous on $\mathbb{R} \backslash D$. Therefore,

$$
\forall t \in \mathbb{R} \backslash D, \quad \mathcal{L}^{1}-\operatorname{ess~sup}_{w \in X} j_{w}(t)=\sup _{w \in X} j_{w}(t)=\sup _{w \in X} \int_{A} u_{t} w d x=J^{* *}\left(u_{t}\right)=J\left(u_{t}\right),
$$

so that

$$
\int_{-\infty}^{+\infty} \mathcal{L}^{1}-\operatorname{esssup}_{w \in X} j_{w}(t) d t=\int_{-\infty}^{+\infty} J\left(u_{t}\right) d t
$$

By 7.21 and 7.22 , the proof of the inequality $J(u) \leq \int_{-\infty}^{+\infty} J\left(u_{t}\right) d t$ is achieved.

- Proof of the inequality $J(u) \geq \int_{\mathbb{R}} J\left(u_{t}\right) d t$. Let us start by showing that, for every $w \in X$, if $\alpha$ is any function in $\mathcal{C}^{\infty}(\mathbb{R},[0,1])$ and $\beta(t):=\int_{0}^{t} \alpha(s) d s$, there holds:

$$
\int_{-\infty}^{+\infty} \alpha(t) j_{w}(t) d t \leq J(\beta \circ u)
$$

Indeed, by applying Fubini's theorem we get

$$
\begin{aligned}
\int_{0}^{+\infty} \alpha(t) j_{w}(t) d t & =\int_{0}^{+\infty} \alpha(t) \int_{A} u_{t}(x) w(x) d x d t=\int_{A} w(x) \int_{0}^{+\infty} \alpha(t) u_{t}(x) d t d x \\
& =\int_{u \geq 0} w(x) \int_{0}^{+\infty} \alpha(t) u_{t}(x) d t d x=\int_{u \geq 0} w(x) \int_{0}^{u(x)} \alpha(t) d t d x \\
& =\int_{u \geq 0} \beta \circ u(x) w(x) d x
\end{aligned}
$$


and

$$
\begin{aligned}
\int_{-\infty}^{0} \alpha(t) j_{w}(t) d t & =\int_{-\infty}^{0} \alpha(t) \int_{A} u_{t}(x) w(x) d x d t=\int_{-\infty}^{0} \alpha(t) \int_{A}\left(u_{t}(x)-1\right) w(x) d x d t \\
& =\int_{A} w(x) \int_{-\infty}^{0} \alpha(t)\left(u_{t}(x)-1\right) d t d x=\int_{u<0} w(x) \int_{-\infty}^{0} \alpha(t)\left(u_{t}(x)-1\right) d t d x \\
& =\int_{u<0} w(x) \int_{u(x)}^{0}(-\alpha(t)) d t d x=\int_{u<0} \beta \circ u(x) w(x) d t
\end{aligned}
$$

Let us remark that, similarly as above, in the computation of the second integral (second equality), we exploited the fact that $w$ has zero integral mean on $A$. The validity of (7.23) readily follows, since

$$
\begin{aligned}
\int_{-\infty}^{+\infty} \alpha(t) j_{w}(t) d t & =\int_{A} \beta \circ u(x) w(x) d x \\
& \leq \sup _{w \in X} \int_{A} \beta \circ u(x) w(x) d x=J^{* *}(\beta \circ u)=J(\beta \circ u) .
\end{aligned}
$$

We are now ready to prove the inequality $J(u) \geq \int_{\mathbb{R}} J\left(u_{t}\right) d t$. We consider the $\mathcal{C}^{\infty}$-convex subset of $L_{\text {loc }}^{1}(A)$ defined by

$$
\mathcal{H}:=\left\{\sum_{i=1}^{k} \alpha_{i} j_{w_{i}}: \alpha_{i} \in \mathcal{C}^{\infty}(\mathbb{R} ;[0,1]), \sum_{i=1}^{k} \alpha_{i} \equiv 1, w_{i} \in X\right\}
$$

For every $u \in L_{\text {loc }}^{1}(A)$, if $v=\sum_{i=1}^{k} \alpha_{i} j_{w_{i}}$ is any function in $\mathcal{H}$, we have

$$
J(u) \geq \sum_{i=1}^{k} J\left(\beta_{i} \circ u\right) \geq \sum_{i=1}^{k} \int_{-\infty}^{+\infty} \alpha_{i}(t) j_{w_{i}}(t) d t=\int_{-\infty}^{+\infty} v(t) d t
$$

where the first inequality holds by assumption (4.28), and the second one by (7.23).

By the arbitrariness of $v \in \mathcal{H}$, by applying the commutation argument between supremum and integral proved in [17, Theorem 1], and recalling the equality $(7.22$, we eventually get

$$
\begin{aligned}
J(u) \geq \sup _{v \in \mathcal{H}} \int_{-\infty}^{+\infty} v d t & =\int_{-\infty}^{+\infty} \mathcal{L}^{1}-\underset{v \in \mathcal{H}}{\operatorname{ess} \sup v(t) d t} \\
& \geq \int_{-\infty}^{+\infty} \mathcal{L}^{1}-\operatorname{ess~sup~}_{w \in X} j_{w}(t) d t=\int_{-\infty}^{+\infty} J\left(u_{t}\right) d t .
\end{aligned}
$$

\section{REFERENCES}

[1] G. Alberti, G. Bouchitté, and G. Dal Maso, The calibration method for the Mumford-Shah functional and free-discontinuity problems, Calc. Var. Partial Differential Equations 16 (2003), no. 3, 299-333.

[2] H. W. Alt and L. A. Caffarelli, Existence and regularity for a minimum problem with free boundary, J. Reine Angew. Math. 325 (1981), 105-144.

[3] H. W. Alt, L. A. Caffarelli, and A. Friedman, Variational problems with two phases and their free boundaries, Trans. Amer. Math. Soc. 282 (1984), no. 2, 431-461.

[4] N. Ambrosio, L. Fusco and D. Pallara, Functions of bounded variation and free discontinuity problems, Oxford Mathematical Monographs, The Clarendon Press Oxford University Press, New York, 2000.

[5] G. Anzellotti, Traces of bounded vector fields and the divergence theorem, Preprint, Uni. Trento.

[6] G. Anzellotti, Pairings between measures and bounded functions and compensated compactness, Ann. Mat. Pura Appl. (4) 135 (1983), 293-318 (1984). 
[7] K.J. Arrow, L. Hurwicz, and H. Uzawa, Studies in linear and non-linear programming, With contributions by H. B. Chenery, S. M. Johnson, S. Karlin, T. Marschak, R. M. Solow. Stanford Mathematical Studies in the Social Sciences, vol. II, Stanford University Press, Stanford, Calif., 1958.

[8] H. Attouch and M. Théra, A general duality principle for the sum of two operators, J. Convex Anal. 3 (1996), no. 1, 1-24.

[9] G. Bouchitté, Convex Analysis and Duality Methods, Encyclopedia of Mathematica Physics, Academic Press (2006), 642-652.

[10] G. Bouchitté and G. Dal Maso, Integral representation and relaxation of convex local functionals on BV $(\Omega)$, Ann. Scuola Norm. Sup. Pisa Cl. Sci. (4) 20 (1993), 483-533.

[11] G. Bouchitté and I. Fragalà, Duality for non-convex variational problems, C. R. Math. Acad. Sci. Paris 353 (2015), no. 4, 375-379.

[12] G. Bouchitté, I. Fragalà, and I. Lucardesi, Shape derivatives for minima of integral functionals, Math. Program. 148 (2014), no. 1-2, Ser. B, 111-142.

[13] G. Bouchitté, I. Fragalà, and I. Lucardesi, A Variational Method for Second Order Shape Derivatives, SIAM J. Control Optim. 54 (2016), no. 2, 1056-1084.

[14] G. Bouchitté, I. Fragalà, and M. Phan, paper in preparation.

[15] G. Bouchitté, C. Galusinski, and M. Phan, paper in preparation.

[16] G. Bouchitté and P.. Suquet, Homogenization, plasticity and yield design, Composite media and homogenization theory (Trieste, 1990), Progr. Nonlinear Differential Equations Appl., vol. 5, Birkhäuser Boston, Boston, MA, 1991, pp. 107-133.

[17] G. Bouchitté and M. Valadier, Integral representation of convex functionals on a space of measures, J. Funct. Anal. 80 (1988), no. 2, 398-420.

[18] S. Boyd and L. Vandenberghe, Convex optimization, Cambridge University Press, Cambridge, 2004.

[19] G. Buttazzo, Semicontinuity, relaxation and integral representation in the calculus of variations, Pitman Research Notes in Mathematics Series, vol. 207, Longman Scientific \& Technical, Harlow, 1989.

[20] L. A. Caffarelli and A. Friedman, Regularity of the boundary of a capillary drop on an inhomogeneous plane and related variational problems, Rev. Mat. Iberoamericana 1 (1985), no. 1, 61-84.

[21] L. A. Caffarelli, D. Jerison, and C. E. Kenig, Global energy minimizers for free boundary problems and full regularity in three dimensions, Noncompact problems at the intersection of geometry, analysis, and topology, Contemp. Math., vol. 350, pp. 83-97.

[22] L. A. Caffarelli and S. Salsa, A geometric approach to free boundary problems, Graduate Studies in Mathematics, vol. 68, American Mathematical Society, Providence, RI, 2005.

[23] P. Cannarsa and C. Sinestrari, Semiconcave functions, Hamilton-Jacobi equations, and optimal control, Progress in Nonlinear Differential Equations and their Applications, 58, Birkhäuser Boston, Inc., Boston, MA, 2004.

[24] A. Chambolle, Convex representation for lower semicontinuous envelopes of functionals in $L^{1}$, J. Convex Anal. 8 (2001), no. 1, 149-170.

[25] G. Dal Maso, Integral representation on $\mathrm{BV}(\Omega)$ of $\Gamma$-limits of variational integrals, Manuscripta Math. $30(1979 / 80)$, no. 4, 387-416.

[26] I. Ekeland and R. Témam, Convex analysis and variational problems, english ed., Classics in Applied Mathematics, vol. 28, Society for Industrial and Applied Mathematics (SIAM), Philadelphia, PA, 1999, Translated from the French.

[27] H. Federer, Real flat chains, cochains and variational problems, Indiana Univ. Math. J. 24 (1974/75), 351-407.

[28] D. Gao, Perfect duality theory and complete solutions to a class of global optimization problems, Optimization 52 (2003), no. 4-5, 467-493, Theory, methods and applications of optimization.

[29] G. Giaquinta, M. Modica and J. Souček, Cartesian currents in the calculus of variations. I, vol. 37, Springer-Verlag, Berlin, 1998.

[30] C. E. Kenig and T. Toro, Free boundary regularity for harmonic measures and Poisson kernels, Ann. of Math. (2) 150 (1999), no. 2, 369-454.

[31] F. Morgan, Calibrations and new singularities in area-minimizing surfaces: a survey, Variational methods (Paris, 1988), Progr. Nonlinear Differential Equations Appl., vol. 4, pp. 329-342.

[32] Y. Nesterov, Introductory Lectures on Convex Optimization, Applied Optimization, Springer US, 2004.

[33] B. Orcan-Ekmekci, On the geometry and regularity of largest subsolutions for a free boundary problem in $\mathbb{R}^{2}$ : elliptic case, Calc. Var. Partial Differential Equations 49 (2014), no. 3-4, 937-962. 
[34] T. Pock, D. Cremers, H. Bischof, and A. Chambolle, An algorithm for minimizing the Mumford-Shah functional, 2009 IEEE 12th International Conference on Computer Vision.

[35] T. Pock, D. Cremers, H. Bischof, and A. Chambolle, Global solutions of variational models with convex regularization, SIAM J. Imaging Sci. 3 (2010), no. 4, 1122-1145.

[36] R. Tyrrell Rockafellar, Convex analysis, Princeton Mathematical Series, No. 28, Princeton University Press, Princeton, N.J., 1970.

[37] A. Visintin, Generalized coarea formula and fractal sets, Japan J. Indust. Appl. Math. 8 (1991), no. 2, $175-201$

(Guy Bouchitté) UfR des Sciences et Techniques, Université de Toulon et du Var, BP 132, 83957 La Garde Cedex (France)

E-mail address: bouchitte@univ-tln.fr

(Ilaria Fragalà) Dipartimento di Matematica, Politecnico di Milano, Piazza Leonardo da Vinci, 32, 20133 Milano (Italy)

E-mail address: ilaria.fragala@polimi.it 